\title{
Normalized solutions for a Schrödinger-Bopp-Podolsky system
}

\author{
Danilo Gregorin Afonso \\ DisSERTATION PRESENTED \\ TO THE \\ Institute of MATHEMATICS AND StATistics \\ OF THE \\ UNIVERSITY OF SÃO PAULO \\ TO \\ OBTAIN THE TITLE \\ $\mathrm{OF}$ \\ MASTER OF SCIENCES \\ Program: Mathematics \\ Advisor: Prof. Dr. Gaetano Siciliano
}

During the development of this work the author was financially supported by CNPq.

São Paulo, January 2020 


\section{Normalized solutions for a Schrödinger-Bopp-Podolsky system}

This version of the dissertation contains the corrections and modifications suggested by the Judging Committee during the defense of the original version of the work, which took place on $14 / 02 / 2020$. A copy of the original version is available at the Institute of Mathematics and Statistics of the University of São Paulo.

Judging Committee:

- Prof. Dr. Gaetano Siciliano (advisor) - IME-USP

- Prof. Dr. Antoine Laurain - IME-USP

- Prof. Dr. Francisco Odair Vieira de Paiva - UFSCar 


\section{Acknowledgements}

To Gaetano, for all he has taught me and for the patience.

To my parents, Ana Laura and Waldemar, for the constant support.

To my mom, again, for her patience.

To Hanna, for her companionship.

To Elvis, Sereno, Bia, Vitor, Carol, Garapa, Felipe, Giovanni and Lissa, for being there.

To all my friends at the Institute, for all the coffees and teas and discussions.

To the Wikipedia and MathStackExchange communities, for obvious reasons.

To CNPq for the financial support. 


\section{Resumo}

GREGORIN AFONSO, D. Soluções normalizadas para um sistema de SchrödingerBopp-Podolsky. 2020. Dissertação (Mestrado) - Instituto de Matemática e Estatística, Universidade de São Paulo, São Paulo, 2020.

Objetivamos estudar um sistema de tipo Schrödinger-Bopp-Podolsky, que consiste de duas equações diferenciais parciais não lineares. Apresentamos um resultado original de existência e multiplicidade de soluções fracas para o problema, ou seja, de existência de pontos críticos de um funcional restrito a uma subvariedade de um espaço de Hilbert. É desenvoldida a teoria do cálculo em espaços de Banach. Discutimos a teoria do gênero de Krasnoselskii e apresentamos o Lema de Deformação e noções correlatas. Discutimos subvariedades em um espaço de Banach e multiplicadores de Lagrange. Provamos a existência e multiplicidade de soluções fracas para o problema proposto.

Palavras-chave: sistema de Schrödinger-Bopp-Podolsky, gênero de Krasnoselskii, Lema de Deformação, multiplicadores de Lagrange, soluções fracas. 


\section{Abstract}

GREGORIN AFONSO, D. Normalized solutions for a Schrödinger-Bopp-Podolsky system. 2020. Dissertation (Masters) - Instituto de Matemática e Estatística, Universidade de São Paulo, São Paulo, 2020.

The aim is to study a Schrödinger-Bopp-Podolsky system of partial differential equations. We present an original result for the existence and multiplicity of weak solutions to the problem, which consists in the determination of critical points for a functional constrained to a submanifold of a Hilbert space. The calculus in Banach spaces is developed. Krasnoselskii's genus theory is discussed, after which the Deformation Lemma and some related notions are presented. Submanifolds of Banach spaces and Lagrange multipliers are discussed. The existence and multiplicity of solutions to the proposed problem is proved.

Keywords: Schrödinger-Bopp-Podolsky system, Krasnoselskii genus, Deformation Lemma, Lagrange multipliers, weak solutions. 


\section{Contents}

$\begin{array}{ll}\text { List of Figures } & \text { ix }\end{array}$

1 Introduction $\quad 1$

2 Calculus in Banach spaces $\quad 3$

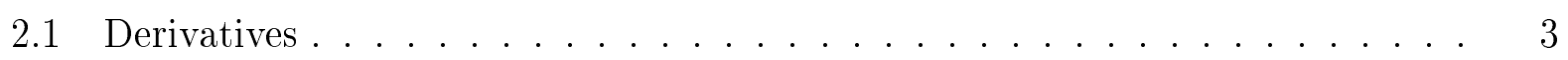

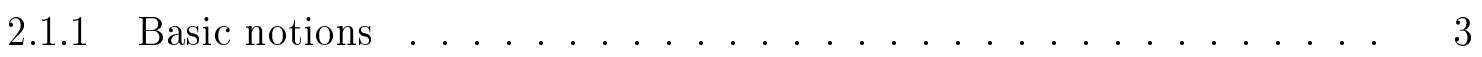

2.1.2 Higher order derivatives . . . . . . . . . . . . . . 9

2.1.3 Partial derivatives . . . . . . . . . . . . . . . . 12

2.2 Local Inversion Theorems . . . . . . . . . . . . . . . 13

2.2.1 The Inverse Function Theorem _. . . . . . . . . . . . . . . . 13

2.2.2 The Implicit Function Theorem . . . . . . . . . . . . . . . . . 18

3 Genus Theory $\quad 21$

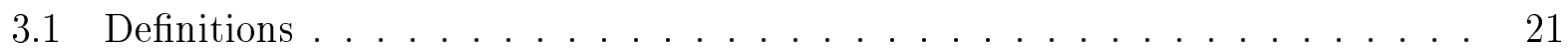

3.2 Properties of the genus . . . . . . . . . . . . . . . . . 22

4 Deformations $\quad 25$

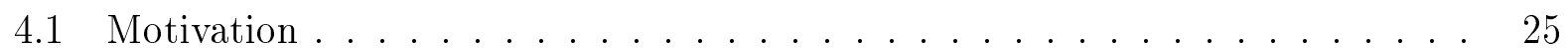

4.2 Preliminary definitions . . . . . . . . . . . . . . . . 27

4.3 The Palais-Smale condition . . . . . . . . . . . . . . 28

4.4 Tangent pseudo-gradient vector fields . . . . . . . . . . . . . . 28

4.5 The Deformation Lemma and its consequences . . . . . . . . . . . . . . 32

4.6 Generalizations . . . . . . . . . . . . . . . 36

5 Submersions, Manifolds, Lagrange Multipliers $\quad 37$

5.1 Submersions and submanifolds . . . . . . . . . . . . . . . . 37

5.2 The Theorem of Lagrange Multipliers . . . . . . . . . . . . . . . . 40

6 Proof of the main result $\quad 43$

6.1 An auxiliary problem . . . . . . . . . . . . . . . 43

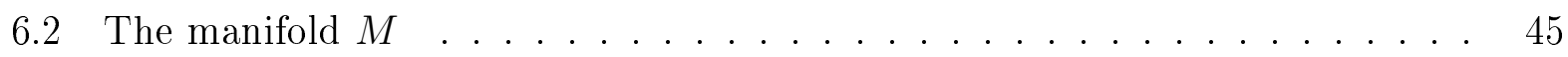

6.3 Existence of solutions . . . . . . . . . . . . . . . 50 
viii CONTENTS

6.4 Multiplicity of solutions . . . . . . . . . . . . . . 56

$\begin{array}{ll}\text { A Useful theorems } & 61\end{array}$

A.1 Neumann boundary value problems . . . . . . . . . . . . . 61

A.2 Sobolev spaces . . . . . . . . . . . . . . . . . 6 61

A.3 The theorem of Borsuk-Ulam _. . . . . . . . . . . . . . . 62

$\begin{array}{ll}\text { Bibliography } & 63\end{array}$

$\begin{array}{ll}\text { Index } & 65\end{array}$ 


\section{List of Figures}

4.1 Graph of $J(x)=x^{3}-3 x \ldots \ldots \ldots \ldots \ldots \ldots$

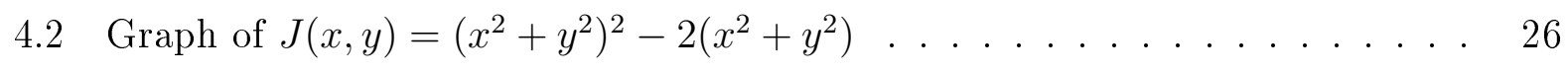

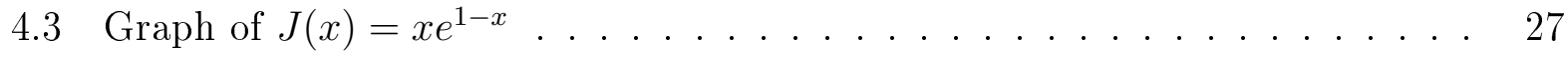


$\mathrm{x}$ LIST OF FIGURES 


\section{Chapter 1}

\section{Introduction}

The Schrödinger-Poisson equation consists of a nonlinear coupling of the Schrödinger equation with a gravitational potential of newtonian form, representing the interaction of a particle with its own gravitational field.

In 1998, Benci and Fortunato (1998) treated a similar problem, where the coupling was with Maxwell's equations and represented the interaction of the particle with its own electromagnetic field. This gives rise to a coupled system of PDEs. In their paper the authors studied the problem with Dirichlet boundary conditions on the unknowns $u$ and $\phi$ and employed variational methods and critical point theory to develop a procedure that would become standard to treat other similar problems.

Later, Pisani and Siciliano (2013) treated a Schrödinger-Poisson system with Neumann boundary conditions on the scalar field $\phi$ and considering the case in which the interaction factor responsible for the coupling of the equations (the $q$ below) is non-constant. This gives rise to important and interesting considerations regarding the geometry of the manifold of possible solutions.

In this dissertation we treat a modification of the problem dealt with by Pisani and Siciliano consisting in the addition of a biharmonic term in the second equation and imposition of appropriate boundary conditions. This can be interpreted as a coupling of Schrödinger equation with Bopp-Podolsky electrodynamics (for more information on this matter, see d'Avenia and Siciliano (2019) and the references therein), although here we focus on the mathematical aspects of the problem.

The aim is to study the following system of partial differential equations in a connected, bounded, smooth open set $\Omega \subset \mathbb{R}^{3}$ :

$$
\begin{gathered}
-\Delta u+q \phi u-\kappa|u|^{p-2} u=\omega u \quad \text { in } \Omega \\
\Delta^{2} \phi-\Delta \phi=q u^{2} \quad \text { in } \Omega
\end{gathered}
$$

where $\omega \in \mathbb{R}$. We assume the following boundary conditions:

$$
\begin{aligned}
& u=0 \quad \text { on } \partial \Omega \\
& \frac{\partial \phi}{\partial \mathbf{n}}=h_{1} \quad \text { on } \quad \partial \Omega \\
& \frac{\partial \Delta \phi}{\partial \mathbf{n}}=h_{2} \quad \text { on } \quad \partial \Omega
\end{aligned}
$$

with $h_{1}, h_{2}$ continuous. The symbol $\mathbf{n}$ denotes the unit vector normal to $\partial \Omega$ pointing outwards. Since $u$ represents the amplitude of the wave function of a particle confined in $\Omega$, we 
assume the following normalizing condition:

$$
\int_{\Omega} u^{2}=1
$$

We also assume that the coupling factor $q$ is continuous on $\bar{\Omega}$ :

$$
q \in C(\bar{\Omega})
$$

Our main theorem is the following:

Theorem 1.0.1. Let

$$
\alpha:=\int_{\partial \Omega} h_{2} d s-\int_{\partial \Omega} h_{1} d s .
$$

Assume that $\inf _{\Omega} q<\alpha<\sup _{\Omega} q$ and that $\left|q^{-1}(\alpha)\right|=0$. Then there exists a solution $(u, \omega, \phi) \in H_{0}^{1}(\Omega) \times \mathbb{R} \times H^{2}(\Omega)$ such that $u \geq 0$. Moreover, there exists infinitely many solutions $\left(u_{n}, \omega_{n}, \phi_{n}\right) \in H_{0}^{1}(\Omega) \times \mathbb{R} \times H^{2}(\Omega)$ to the problem (1.1) - (1.6), with

$$
\int_{\Omega}\left|\nabla u_{n}\right|^{2} d x \rightarrow+\infty
$$

The motivation for the definition of $\alpha$ and the importance of the hypothesis will be made clear in Chapter 6.

In our computations, nothing important is lost if we drop the term $\kappa|u|^{p-2} u$, so we make this simplification. One only would have to take account of the appropriate values of $p$. See Remark 6.4.3.

It is now time to fix some notation. In what follows, $X, Y$ and eventually $Z$ will denote Banach spaces. Usually, $U \subset X$ will be an open set. The symbol $\mathcal{L}(X, Y)$ denotes the set of continuous linear operators $T: X \longrightarrow Y$. The value of $T$ at $x \in X$ will be denoted by $T[x]$. The letter $c$ denotes a constant whose value can change from line to line. 


\section{Chapter 2}

\section{Calculus in Banach spaces}

\subsection{Derivatives}

\subsubsection{Basic notions}

Recall from calculus in finite dimension the meaning of "derivative" of a function at some given point. The idea was to obtain for the given function a linear approximation for the function in some neighborhood of the point. In one dimension we had the angular coefficient of the tangent line; in $N$ dimensions we had the matrix of the linear transformation. The concept extends immediately to infinitely many dimensions:

Definition 2.1.1. Let $X, Y$ be Banach spaces and $U \subset X$ be an open set. We say that $f: U \longrightarrow Y$ is Fréchet differentiable(differentiable for short) at the point $u \in U$ if there exists $L_{u} \in \mathcal{L}(X, Y)$ such that

$$
f(u+h)-f(u)=L_{u}[h]+R(h),
$$

with

$$
\lim _{\|h\| \rightarrow 0} \frac{\|R(h)\|}{\|h\|}=0,
$$

that is, $R(h)=o(h)^{1}$. The map $L_{u}$ is the differential of $f$ at $u$.

Remark 2.1.2. The definition of Fréchet differentiablity depends only on the topology of $X$ and $Y$ and not on the specific norms these spaces are endowed with.

This definition was first introduced by Fréchet ${ }^{2}$ in 1911.

Of course, all generalizations must reduce to the original cases. It is easy to see that the definition above reduces to the finite dimensional case if the spaces $X$ and $Y$ are taken to be $\mathbb{R}$ or $\mathbb{R}^{N}$.

We remark that the differential is unique. This is an immediate consequence of the following

Lemma 2.1.3. Suppose $L_{1}, L_{2} \in \mathcal{L}(X, Y)$ are such that $L_{1}[h]-L_{2}[h]=o(h)$. Then $L_{1}=L_{2}$.

Proof. By hypothesis, we have that

$$
\frac{\left\|L_{1}[h]-L_{2}[h]\right\|}{\|h\|} \stackrel{\|h\| \rightarrow 0}{\longrightarrow} 0 .
$$

${ }^{1}$ This is known as little-o notation.

${ }^{2}$ Maurice Fréchet. 
Now, suppose the Lemma to be false. Then there is some $h^{*}$ such that $L_{1}\left[h^{*}\right]-L_{2}\left[h^{*}\right] \neq 0$. Now, letting $h=t h^{*}$ we have

$$
\lim _{t \rightarrow 0} \frac{\left\|L_{1}\left[t h^{*}\right]-L_{2}\left[t h^{*}\right]\right\|}{\left\|t h^{*}\right\|}=\lim _{t \rightarrow 0} \frac{t|| L_{1}\left[h^{*}\right]-L_{2}\left[h^{*}\right] \|}{t|| h^{*} \|} \neq 0
$$

a contradiction.

The uniqueness of the differential compels us to adopt the following

Notation. Let $f: X \longrightarrow Y$ be a map differentiable at $u \in X$. Then the differential of $f$ at $u$ is the continuous linear transformation denoted by

$$
f^{\prime}(u) \text { or sometimes by } d f(u) \text {. }
$$

Remark 2.1.4. If $f$ is differentiable at $u \in U$ then it is continuous at $u$. Indeed,

$$
f(u+h)-f(u)=f^{\prime}(u)[h]+o(h) \stackrel{h \rightarrow 0}{\longrightarrow} 0 .
$$

Definition 2.1.5. If a function $f$ is differentiable at all $u \in U$, we say that it is differentiable in $U$. The map

$$
\begin{aligned}
f^{\prime}: U & \longrightarrow \mathcal{L}(X, Y) \\
u & \longmapsto f^{\prime}(u)
\end{aligned}
$$

is the derivative of $f$. If $f^{\prime}$ is continuous, we say that $f$ is of class $C^{1}$.

Remark 2.1.6. Note the difference between the concepts of differential and derivative. The differential is a linear approximation for the function in the neighborhood of a given point, while the derivative is a map that associates to each point a linear map.

We now see some examples.

Example 2.1.7. $f$ is constant $\Longrightarrow f^{\prime}(u)=0$ for all $u \in U$.

Example 2.1.8. If $f \in \mathcal{L}(X, Y)$ then $f^{\prime}(u)=f$ for all $u \in U$. Indeed, in this case

$$
f(u+h)-f(u)=f(h),
$$

from which the claim immediately follows.

Example 2.1.9. Let $b: X \times Y \longrightarrow Z$ be a continuous bilinear form. Then $b$ is differentiable at any $(u, v) \in X \times Y$ and $d b(u, v)$ is the linear map

$$
(h, k) \mapsto b(u, k)+b(h, v)
$$

Indeed, note that

$$
b(u+h, v+k)-b(u, v)=b(u, k)+b(h, v)+b(h, k)
$$

but by continuity we have (see Brezis (2010), pp. 138)

$$
\|b(h, k)\| \leq c\|h\|\|k\|
$$

and of course

$$
\lim _{(h, k) \rightarrow 0} \frac{c\|h\|\|k\|}{\|h\|+\|k\|}=0,
$$


from which the claim follows.

Example 2.1.10. Let $b: X \times X \longrightarrow \mathbb{R}$ be a continuous bilinear form. Define

$$
\begin{aligned}
f: X & \longrightarrow \mathbb{R} \\
u & \longmapsto b(u, u)
\end{aligned}
$$

Then

$$
f^{\prime}(u)=b(u, \cdot)+b(\cdot, u) .
$$

Of course, if $b$ is symmetric then $f^{\prime}(u)=2 b(u, \cdot)$. What if $X$ is a Hilbert space and $b(u, v)=(u \mid v)$, the scalar product? Then $f(u)=\|u\|^{2}$ and $f^{\prime}(u)=2(u \mid \cdot)$.

Example 2.1.11. Let $\Omega$ be a smooth open set in $\mathbb{R}^{N}, A=A(x)$ be an $N \times N$ symmetric matrix with $a_{i j} \in L^{\infty}(\Omega)$ and let $q \in L^{\infty}(\Omega)$. Then

$$
J(u)=\frac{1}{2} \int_{\Omega}(A \nabla u) \cdot \nabla u d x+\int_{\Omega} q u^{2} d x
$$

defines a functional on the Sobolev space $H^{1}(\Omega)^{3}$ whose Fréchet derivative is given by

$$
J^{\prime}(u)[v]=\int_{\Omega}(A \nabla u) \cdot \nabla v d x+2 \int_{\Omega} q u v d x .
$$

Indeed, note that $J$ is the functional induced by the following bilinear form on $H_{0}^{1}(\Omega) \times H_{0}^{1}(\Omega)$ :

$$
b(u, v)=\frac{1}{2} \int_{\Omega}(A \nabla u) \cdot \nabla v d x+2 \int_{\Omega} q u v d x .
$$

This completes the proof.

The usual rules of calculus remain true for the Fréchet derivative.

Proposition 2.1.12. Let $f, g$ be differentiable functions at $u \in X$ and let $a, b \in \mathbb{R}$. Then $a f+b g$ is differentiable at $u$ and

$$
(a f+b g)^{\prime}(u)=a f^{\prime}(u)+b g^{\prime}(u) .
$$

Proof. Note that

$$
(a f+b g)(u+h)-(a f+b g)(u)-a f^{\prime}(u)[h]-b g^{\prime}(u)[h]=o(h),
$$

from which the claim immediately follows.

Proposition 2.1.13 (Chain Rule). Let $X, Y$ and $Z$ be Banach spaces, $u \in X$ and $f: X \longrightarrow$ $Y, g: Y \longrightarrow Z$ such that $f$ is differentiable at $u$ and $g$ is differentiable at $v=f(u)$. Then

$$
g \circ f: X \longrightarrow Z
$$

is differentiable at $u$ and

$$
(g \circ f)^{\prime}(u)=g^{\prime}(f(u)) f^{\prime}(u)
$$

\footnotetext{
${ }^{3}$ See Brezis (2010).
} 
Proof. We have

$$
\begin{aligned}
& f(u+h)=f(u)+f^{\prime}(u)[h]+o(h), \\
& g(v+k)=g(v)+g^{\prime}(v)[k]+o(k) .
\end{aligned}
$$

Then

$$
\begin{aligned}
g(f(u+h))-g(f(u)) & =g\left(f(u)+f^{\prime}(u)[h]+o(h)\right)-g(f(u)) \\
& =g^{\prime}(f(u))\left[f^{\prime}(u)[h]+o(h)\right]+o\left(f^{\prime}(u)[h]+o(h)\right) \\
& =g^{\prime}(f(u))\left[f^{\prime}(u)[h]\right]+g^{\prime}(f(u))[o(h)]+o\left(f^{\prime}(u)[h]+o(h)\right) .
\end{aligned}
$$

We must show that the two last terms are $o(h)$. That

$$
\frac{g^{\prime}(f(u))[o(h)]}{\|h\|} \stackrel{h \rightarrow 0}{\longrightarrow} 0
$$

is clear by linearity and continuity of $g^{\prime}(f(u))$. Now, note that

$$
\frac{o\left(f^{\prime}(u)[h]+o(h)\right)}{\|h\|}=\frac{o\left(f^{\prime}(u)[h]+o(h)\right)}{\|h\|} \frac{\left\|o\left(f^{\prime}(u)[h]+o(h)\right)\right\|}{\left\|o\left(f^{\prime}(u)[h]+o(h)\right)\right\|} .
$$

But

$$
\begin{aligned}
\frac{\left\|o\left(f^{\prime}(u)[h]+o(h)\right)\right\|}{\|h\|} & =\left\|f^{\prime}(u)\left[\frac{h}{\|h\|}\right]+\frac{o(h)}{\|h\|}\right\| \\
& \leq\left\|f^{\prime}(u)\right\|+\frac{o(h)}{\|h\|} \\
& \leq C
\end{aligned}
$$

near $h=0$. Since $f^{\prime}(u)[h]+o(h) \stackrel{h \rightarrow 0}{\longrightarrow} 0$, we have that

$$
\frac{\left\|o\left(f^{\prime}(u)[h]+o(h)\right)\right\|}{\|h\|} \stackrel{h \rightarrow 0}{\longrightarrow} 0
$$

which completes the proof.

Just like in the case of $R^{N}$, there is a concept of directional derivative. It was introduced by Gateaux ${ }^{4}$ in 1913.

Definition 2.1.14. Let $f: X \longrightarrow Y$. Then $f$ is said to be Gateaux-differentiable (Gdifferentiable for short) at $u \in X$ if there exists $A \in \mathcal{L}(X, Y)$ such that for all $h \in X$ there exists

$$
\lim _{t \rightarrow 0^{+}} \frac{f(u+t h)-f(u)}{t}:=A[h] .
$$

The map $A$ is uniquely determined (this follows by the argument in Lemma 2.1.3) and will be denoted by $f_{G}^{\prime}(u)$.

It is immediate that Fréchet differentiability implies Gateaux differentiability. However, the converse is not true: Gateaux differentiability doesn't even imply continuity. Consider

${ }^{4}$ René Gateaux. 
the following example in $\mathbb{R}^{2}$ :

$$
\begin{aligned}
& F(x, y)=\left(\frac{x^{2} y}{x^{4}+y^{2}}\right)^{2}, \quad y \neq 0, \\
& F(x, 0)=0
\end{aligned}
$$

Notice that $F((\sqrt{y}, y))=1 / 2$ but $F(0,0)=0$.

We now prove the so-called Mean-Value Inequality. It plays a fundamental role in what follows.

Notation. Given $u, v \in U$ the segment between them is denoted by

$$
[u, v]:=\{t u+(1-t) v: t \in[0,1]\} .
$$

Theorem 2.1.15 (Mean Value Inequality). Let $f: U \longrightarrow Y$ be G-differentiable at any point of the open set $U \subset X$. Given $u, v$ such that $[u, v] \subset U$ it holds that

$$
\|f(u)-f(v)\| \leq \sup _{w \in[u, v]}\left\|f_{G}^{\prime}(w)\right\|\|u-v\| .
$$

Proof. If $f(u)=f(v)$ the claim is trivial. Suppose then $f(u) \neq f(v)$. By a corollary of the Hahn-Banach theorem ${ }^{5}$ there exists $\psi \in Y^{*}$ such that

$$
\|\psi\|=1
$$

and

$$
\psi(f(u)-f(v))=\|f(u)-f(v)\|
$$

For $t \in[0,1]$ let

$$
\gamma(t)=t u+(1-t) v
$$

and consider the map $h:[0,1] \longrightarrow \mathbb{R}$ defined by

$$
h(t)=\psi(f(\gamma(t)) .
$$

Notice that

$$
\gamma(t+\tau)=\gamma(t)+\tau(u-v)
$$

From this it follows that

$$
\frac{h(t+\tau)-h(t)}{\tau}=\psi\left(\frac{f(\gamma(t)+\tau(u-v))-f(\gamma(t))}{\tau}\right)
$$

Passing to the limit as $\tau \rightarrow 0$ we have

$$
h^{\prime}(t)=\psi\left(f_{G}^{\prime}(\gamma(t))[u-v]\right) .
$$

Now, by the classic Mean-Value Theorem we have that $h(1)-h(0)=h^{\prime}(\theta)$ for some $\theta \in(0,1)$.

\footnotetext{
${ }^{5}$ See Theorem 12.2 in Bachman and Narici (2000)
} 
Now, note that

$$
\begin{aligned}
\|f(u)-f(v)\| & =h(1)-h(0) \\
& =h^{\prime}(\theta) \\
& =\psi\left(f_{G}^{\prime}(\theta u+(1-\theta v))[u-v]\right) \\
& \leq\|\psi\|\left\|f_{G}^{\prime}(\theta u+(1-\theta) v)\right\|\|u-v\|
\end{aligned}
$$

But recall that $\|\psi\|=1$ and that $\theta u+(1-\theta) v \in[u, v]$. This completes the proof.

There is a well-known result in classic Calculus that says that if a function has continuous partial derivatives at a point then it is differentiable at the given point. We have an analogous relation between the Gateaux and Fréchet derivatives.

Proposition 2.1.16. Let $f: U \longrightarrow Y$ be a G-differentiable function in $U$ and suppose $f_{G}^{\prime}$ to be continuous at $u \in U$. Then $f$ is differentiable at $u$ and $f^{\prime}(u)=f_{G}^{\prime}(u)$.

Proof. The proposition gives us a hint to the proof: it suffices to show that

$$
R(h)=f(u+h)-f(u)-f_{G}^{\prime}(u)[h]
$$

is $o(h)$.

Note that $R(h)$ is G-differentiable for $h$ small enough:

$$
\begin{aligned}
\frac{R(h+t k)-R(h)}{t} & =\frac{1}{t}\left(f(u+h+t k)-f_{G}^{\prime}(u)[h+t k]-f(u+h)+f_{G}^{\prime}(u)[h]\right) \\
& =\frac{f(u+h+t k)-f(u+h)}{t}-f_{G}^{\prime}[k] \\
& \rightarrow f_{G}^{\prime}(u+h)[k]-f_{G}^{\prime}(u)[k] .
\end{aligned}
$$

( $h$ must be small enough so that $u+h \in U$ ).

Note that $R(0)=0$. We can then apply the Mean Value Inequality on the segment $[0, h]$ to obtain

$$
\|R(h)\| \leq \sup _{0 \leq t \leq 1}\left\|R_{G}^{\prime}(t h)\right\|\|h\| .
$$

But, as we have seen, $R_{G}^{\prime}(h)=f_{G}^{\prime}(u+h)-f_{G}^{\prime}(u)$. Thus

$$
\frac{\|R(h)\|}{\|h\|} \leq \sup _{0 \leq t \leq 1}\left\|f_{G}^{\prime}(u+t h)-f_{G}^{\prime}(u)\right\| \stackrel{h \rightarrow 0}{\longrightarrow} 0,
$$

since $f_{G}^{\prime}$ is continuous. This completes the proof.

Remark 2.1.17. Computing Fréchet derivatives may be difficult, but Gateaux derivatives are more treatable. In view of this, Proposition 2.1.16 provides a method to find Fréchet derivatives: one computes the Gateaux derivative and then show that it is continuous.

The next example will be useful afterwards.

Example 2.1.18. Let $\Omega$ be a smooth, bounded open set and $q \in C(\bar{\Omega})$. The map

$$
u \in L^{6}(\Omega) \mapsto q u^{2} \in L^{6 / 5}(\Omega)
$$


is of class $C^{1}$. Indeed:

$$
\lim _{t \rightarrow 0^{+}} \frac{f(u+t h)-f(u)}{t}=\lim _{t \rightarrow 0^{+}} u h+t h^{2}=u h .
$$

Hence $f_{G}^{\prime}(u)[h]=u h$. Let us show that $f_{G}^{\prime}$ is continuous with respect to $u$. Let $u_{n} \rightarrow u$ in $L^{6}(\Omega)$. Then

$$
\left\|f_{G}^{\prime}(u)-f_{G}^{\prime}\left(u_{n}\right)\right\|=\sup _{\|h\|_{6}=1}\left\|\left(u-u_{n}\right) h\right\|_{6 / 5} \leq\left\|u-u_{n}\right\|_{6 / 4} \rightarrow 0
$$

by Hölder's inequality, which completes the proof.

We close this section with an example that will be important in Section 2.1.3.

Example 2.1.19. Let $f \in C([a, b], Y)$ and $\operatorname{let}^{6}$

$$
F(t)=\int_{a}^{t} f(\xi) d \xi
$$

Then $F$ is differentiable and $F^{\prime}(t)=f(t)$. Indeed, we have

$$
\frac{F(t+h)-F(t)}{h}=\frac{1}{h} \int_{t}^{t+h} f(\xi) d \xi
$$

But

$$
\int_{t}^{t+h} f(t) d \xi=h f(t)
$$

Then we have

$$
\begin{aligned}
\frac{F(t+h)-F(t)}{h}-f(t) & =\frac{1}{h}\left[\int_{t}^{t+h} f(\xi) d \xi-\int_{t}^{t+h} f(t) d \xi\right] \\
& =\frac{1}{h} \int_{t}^{t+h}(f(\xi)-f(t)) d \xi
\end{aligned}
$$

Hence,

$$
\left\|\frac{F(t+h)-F(t)}{h}-f(t)\right\| \leq \frac{1}{|h|}|h| \sup _{[t, t+h]}\|f(\xi)-f(t)\| \rightarrow 0 .
$$

which completes the proof.

\subsubsection{Higher order derivatives}

Definition 2.1.20. Let $f \in C(U, Y)$ (where, as always, $U \subset X$ is an open subset) be a differentiable function. If the map

$$
f^{\prime}: U \longrightarrow \mathcal{L}(X, Y)
$$

is differentiable at $u \in U$ then $f$ is said to be twice Fréchet differentiable at $u$. The second differential of $f$ at $u$ is

$$
f^{\prime \prime}(u)=\left(f^{\prime}\right)^{\prime}(u)
$$

\footnotetext{
${ }^{6}$ The theory of integration works just like for functions into $\mathbb{R}^{N}$. See Lang (1997) for details.
} 
Higher order differentials are defined in an analogous manner:

$$
f^{(k)}(u)=\left(f^{(k-1)}\right)^{\prime}(u) .
$$

Note that

$$
f^{\prime \prime}(u) \in \mathcal{L}(X, \mathcal{L}(X, Y))
$$

Indeed, $f^{\prime}$ takes values in $\mathcal{L}(X, Y)$.

It is useful, however, to see $f^{\prime \prime}(u)$ as a bilinear map. Recall that we can identify $\mathcal{L}(X, \mathcal{L}(X, Y))$ with $\mathcal{L}_{2}(X, Y)$, the space of continuous bilinear forms from $X \times X$ to $Y$. Indeed, given $A \in \mathcal{L}(X, \mathcal{L}(X, Y))$ let $\Phi_{A}$ be the bilinear form

$$
\Phi_{A}\left(u_{1}, u_{2}\right)=\left[A\left(u_{1}\right)\right]\left(u_{2}\right)
$$

Of course $\Phi_{A}$ is continuous, since both $A$ and $A\left(u_{1}\right)$ are continuous. Conversely, any $\Phi \in$ $\mathcal{L}_{2}(X, Y)$ defines a map

$$
\begin{aligned}
\phi: X & \longrightarrow \mathcal{L}(X, Y) \\
h & \longmapsto \Phi(h, \cdot)
\end{aligned}
$$

Note that we have just defined an isomorphism between $\mathcal{L}(X, \mathcal{L}(X, Y))$ and $\mathcal{L}_{2}(X, Y)$. Moreover,

$$
\begin{aligned}
\|\phi\|_{\mathcal{L}(X, \mathcal{L}(X, Y))} & =\sup _{\|h\| \leq 1}\|\Phi(h)\|_{\mathcal{L}(X, Y)} \\
& =\sup _{\|h\| \leq 1} \sup _{\|k\| \leq 1}\|\Phi(h, k)\| \\
& =\|\Phi\|_{\mathcal{L}_{2}(X, Y)}
\end{aligned}
$$

and thus we actually have an isometry.

From now on, $f^{\prime \prime}(u)$ will be viewed as the bilinear form just defined. Its value at a pair $(h, k)$ will be denoted

$$
f^{\prime \prime}(u)[h, k]
$$

Definition 2.1.21. If $f$ is twice differentiable at every $u \in U$ we say that $f^{\prime \prime}: U \longrightarrow$ $\mathcal{L}_{2}(X, Y)$ is the second derivative of $f$. Higher order derivatives are defined in an analogous manner.

Proposition 2.1.22. Let $f \in C^{2}(U, Y)$. Then $f^{\prime \prime}(u)$ is symmetric for all $u \in U$.

Proof. Let $\varphi \in Y^{*}$. Define

$$
g:(t, s) \in \mathbb{R}^{2} \mapsto \varphi(f(u+t v+s w)) \in \mathbb{R}
$$

By the Chain Rule we have that

$$
\begin{aligned}
& \frac{\partial g}{\partial t}(t, s)=\varphi\left(f^{\prime}(u+t v+s w)[v]\right) \\
& \frac{\partial}{\partial s}\left(\frac{\partial g}{\partial t}\right)(t, s)=\varphi\left(f^{\prime \prime}(u+t v+s w)[v, w]\right)
\end{aligned}
$$

Analogously,

$$
\frac{\partial}{\partial t}\left(\frac{\partial g}{\partial s}\right)(t, s)=\varphi\left(f^{\prime \prime}(u+t v+s w)[w, v]\right)
$$


Now, since $g$ is of class $C^{2}$ (it is a composition of $C^{2}$ maps), it follows that

$$
\varphi\left(f^{\prime \prime}(u+t v+s w)[v, w]\right)=\varphi\left(f^{\prime \prime}(u+t v+s w)[w, v]\right)
$$

The equality holds for all $\varphi \in Y^{*}$, thus

$$
f^{\prime \prime}(u+t v+s w)[v, w]=f^{\prime \prime}(u+t v+s w)[w, v]
$$

for all $t, s$. In particular, for $t=s=0$ we get

$$
f^{\prime \prime}(u)[v, w]=f^{\prime \prime}(u)[w, v]
$$

and the proof is complete.

An analogous theorem holds for higher order derivatives. See Lang (1997) for details.

Just like in classic Calculus, second derivatives are very useful for the study of extrema of functionals. For an extensive discussion, see Blanchard and Brüning (1992).

If $f^{(k)}: U \longrightarrow Y$ is a continuous map we say that $f \in C^{k}(U, Y)$.

We can now generalize Taylor's formula.

Theorem 2.1.23 (Taylor's formula). Let $f: U \longrightarrow Y$ be a map of class $C^{n}$ defined in an open, convex neighborhood of $u$. Then

$$
f(u+h)=f(u)+\sum_{j=1}^{n-1} \frac{1}{j !} f^{(j)}(u)[h]^{j}+R_{n}
$$

with

$$
R_{n}=\int_{0}^{1} \frac{(1-t)^{n-1}}{(n-1) !} f^{(n)}(u+t h) h^{n} d t .
$$

Here, $[h]^{j}=[h, \ldots, h] j$ times.

Proof. For $t \in[0,1]$ and $u, h \in U$ let $\gamma(t)=u+t h$ and

$$
\begin{aligned}
\phi:[0,1] & \longrightarrow Y \\
t & \longmapsto f(\gamma(t)) .
\end{aligned}
$$

Then $\phi$ is of class $C^{n}$ and

$$
\begin{aligned}
& \phi^{\prime}(t)=f^{\prime}(u+t h)[h], \\
& \phi^{\prime \prime}(t)=f^{\prime \prime}(u+t h)[h]^{2}, \\
& \vdots \\
& \phi^{(n)}(t)=f^{(n)}(u+t h)[h]^{n} .
\end{aligned}
$$

But then

$$
\phi(1)=\phi(0)+\phi^{\prime}(0)+\ldots+\frac{1}{(n-1) !} \phi^{(n)}(0)+\frac{1}{(n-1) !} \int_{0}^{1}(1-t)^{n-1} \phi^{(n)}(t) d t,
$$

and hence the formula follows.

For more details on higher order derivatives, see Lang (1997) and Ambrosetti and Prodi (1993). 


\subsubsection{Partial derivatives}

The formulation of partial derivatives is as simple as in classic Calculus.

Consider a product of Banach spaces:

$$
X=X_{1} \times \ldots \times X_{n}
$$

and let $U_{i} \subset X_{i}$ be open subset. Denote $U=U_{1} \times \ldots \times U_{n}$ and let

$$
f: U \longrightarrow Y
$$

be a map. We can write elements $u \in U$ in terms of their coordinates: $u=\left(u_{1}, \ldots, u_{n}\right)$.

Definition 2.1.24. Let $u_{1}, \ldots, u_{i-1}, u_{i+1}, \ldots u_{n}$ be fixed. If the map

$$
u_{i} \mapsto f\left(u_{1}, \ldots, u_{n}\right)
$$

is differentiable in $U$ then its derivative is the partial derivative of $f$ in the $\mathrm{i}$-th coordinate, and its value at $u \in U$ is denoted by $D_{i} f(u)$ or $f_{u_{i}}^{\prime}(u)$.

Note that for each $u \in U$ we have

$$
D_{i} f(u) \in \mathcal{L}\left(X_{i}, Y\right) .
$$

Just as in Calculus in finite dimension, smoothness is equivalent to smoothness of the partial derivatives:

Proposition 2.1.25. Let $U_{i} \subset X_{i}$ be open sets and let $f: U=\Pi_{1}^{N} U_{i} \longrightarrow Y$. Then $f$ is of class $C^{k}$ if and only if each partial derivative

$$
D_{i} f: U \longrightarrow \mathcal{L}\left(X_{i}, Y\right)
$$

is of class $C^{k-1}$. If this is the case, then

$$
f^{\prime}(u)[h]=\sum_{1}^{n} D_{i} f(u)\left[h_{i}\right]
$$

for $h=\left(h_{1}, \ldots, h_{n}\right) \in \Pi_{1}^{n} X_{i}$

Proof. It suffices to write a proof for $n=2$. For greater $n$, the argument is exactly the same.

First, suppose the partial derivatives exist and are continuous. Let $(u, v) \in U$ and $h=$ $\left(h_{1}, h_{2}\right)$. Then

$$
\begin{aligned}
f\left(u+h_{1}, v+h_{2}\right) & -f(u, v) \\
& =f\left(u+h_{1}, v+h_{2}\right)-f\left(u+h_{1}, v\right)+f\left(u+h_{1}, v\right)-f(u, v) \\
& =\int_{0}^{1} D_{2} f\left(u+h_{1}, v+t h_{2}\right)\left[h_{2}\right] d t+\int_{0}^{1} D_{1} f\left(u+t h_{1}, v\right)\left[h_{1}\right] d t .
\end{aligned}
$$

Let

$$
\psi\left(h_{1}, t h_{2}\right):=D_{2} f\left(u+h_{1}, v+t h_{2}\right)-D_{2} f(u, v)
$$


The first integral can be written as

$$
\begin{aligned}
\int_{0}^{1} D_{2} f\left(u+h_{1}, v+t h_{2}\right)\left[h_{2}\right] d t & =\int_{0}^{1} D_{2} f(u, v)\left[h_{2}\right] d t+\int_{0}^{1} \psi\left(h_{1}, t h_{2}\right)\left[h_{2}\right] d t \\
& =D_{2} f(u, v)\left[h_{2}\right]+\int_{0}^{1} \psi\left(h_{1}, t h_{2}\right)\left[h_{2}\right] d t
\end{aligned}
$$

Now, note that

$$
\begin{aligned}
\left|\int_{0}^{1} \psi\left(h_{1}, t h_{2}\right)\left[h_{2}\right] d t\right| & \leq \sup _{t \in[0,1]}\left\|\psi\left(h_{1}, t h_{2}\right)\right\|\left\|h_{2}\right\| \\
& \leq\|h\| \sup _{t \in[0,1]}\left\|\psi\left(h_{1}, t h_{2}\right)\right\| \\
& =o(h)
\end{aligned}
$$

since, by continuity,

$$
\psi\left(h_{1}, t h_{2}\right)=D_{2} f\left(u+h_{1}, v+t h_{2}\right)-D_{2} f(u, v) \stackrel{h \rightarrow 0}{\longrightarrow} 0 .
$$

Now let

$$
\phi\left(t h_{1}, h_{2}\right)=D_{1} f\left(u+t h_{1}, v\right)-D_{1} f(u, v) .
$$

We have

$$
\begin{aligned}
\int_{0}^{1} D_{1} f\left(u+t h_{1}, v\right)\left[h_{1}\right] d t & =\int_{0}^{1} D_{1} f(u, v)\left[h_{1}\right] d t+\int_{0}^{1} \phi\left(t h_{1}, h_{2}\right) d t \\
& =D_{1} f(u, v)\left[h_{1}\right]+\int \phi\left(t h_{1}, h_{2}\right) d t
\end{aligned}
$$

Estimating the error as we have just done we conclude that

$$
f\left(u+h_{1}, v+h_{2}\right)-f(u, v)=D_{1} f(u, v)\left[h_{1}\right]+D_{2} f(u, v)\left[h_{2}\right]+o(h),
$$

hence $f$ is differentiable and the derivative is given as above.

To prove the converse statement one must only evaluate the derivative along the directions $\left(h_{1}, 0\right)$ and $\left(0, h_{2}\right)$.

\subsection{Local Inversion Theorems}

One of the most important theorems in classic Calculus is the Inverse Function Theorem, together with its cousin Implicit Function Theorem. In this chapter we will see how they generalize to infinite-dimensional spaces.

We follow Ambrosetti and Prodi (1993) and Lang (1995).

\subsubsection{The Inverse Function Theorem}

Definition 2.2.1. Let $X$ and $Y$ be Banach spaces. A map $A \in \mathcal{L}(X, Y)$ is said to be invertible if there exists $A^{-1} \in \mathcal{L}(Y, X)$ such that

$$
A^{-1} \circ A=I_{X}, \quad A \circ A^{-1}=I_{Y} .
$$


We set

$$
\operatorname{Inv}(X, Y):=\{A \in \mathcal{L}(X, Y): A \text { is invertible }\} .
$$

In order to prove our next proposition we need a preliminary lemma:

Lemma 2.2.2. Let $A \in \mathcal{L}(X, X)$ such that $\|A\|<1$. Then $I-A$ is invertible and its inverse is given by the convergent series

$$
(I-A)^{-1}=\sum_{0}^{\infty} A^{n}
$$

Proof. Note that $\|A A\| \leq\|A\|\|A\|$, thus $\left\|A^{n}\right\| \leq\|A\|^{n}$. Then

$$
\left\|I+A+\ldots+A^{n}\right\| \leq 1+\|A\|+\ldots+\|A\|^{n}
$$

which converges, since $\|A\|<1$ (it is the geometric series). Thus the series really converges. Now, note that

$$
(I-A)\left(I+A+\ldots+A^{n}\right)=I-A^{n+1}=\left(I+A+\ldots+A^{n}\right)(I-A)
$$

and that $A^{n} \rightarrow 0$. Taking the limit completes the proof.

The above lemma shows that there is a ball of radius one around the identity of invertible operators. Indeed, if $\|I-A\|<1$ then $A=I-(I-A)$ is invertible.

Proposition 2.2.3. If $A \in \operatorname{Inv}(X, X)$ then every $T \in \mathcal{L}(X, X)$ such that

$$
\|T-A\|<\frac{1}{\left\|A^{-1}\right\|}
$$

is invertible. Hence, $\operatorname{Inv}(X, X)$ is a open subset of $\mathcal{L}(X, X)$.

Proof. Note that

$$
\left\|T A^{-1}-I\right\|=\left\|(T-A) A^{-1}\right\| \leq\left\|A^{-1}\right\|\|T-A\| \leq 1 .
$$

Thus $T A^{-1}$ is invertible. Hence, $T=T A^{-1} A$ is invertible.

Proposition 2.2.4. The map

$$
\varphi: A \in \operatorname{Inv}(X, X) \mapsto A^{-1} \in \mathcal{L}(X, X)
$$

is infinitely differentiable, with

$$
\varphi^{\prime}(A)[B]=-A^{-1} \circ B \circ A^{-1} .
$$

Proof. For $B$ sufficiently small we have that

$$
\begin{aligned}
(A+B)^{-1}-A^{-1} & =\left(A\left(I+A^{-1} B\right)\right)^{-1}-A^{-1} \\
& =\left[\left(I+A^{-1} B\right)^{-1}-I\right] A^{-1} .
\end{aligned}
$$

By the above lemma, if $B$ is so small that $\left\|A^{-1} B\right\|<1$ then

$$
\left(I+A^{-1} B\right)^{-1}=I-A^{-1} B+\left(A^{-1} B\right)^{2} g(B)
$$


where $g$ is a convergent power series. Hence

$$
(A+B)^{-1}-A^{-1}=-A^{-1} B A^{-1}+\left(A^{-1} B\right)^{2} g(B) A^{-1} .
$$

Now, notice that the series is bounded, because it is convergent. Then the second term above can be estimated:

$$
\left\|\left(A^{-1} B\right)^{2} g(B) A^{-1}\right\| \leq C\|B\|^{2} .
$$

Thus

$$
\varphi^{\prime}(A)[B]=-A^{-1} \circ B \circ A^{-1} .
$$

That $\varphi$ is infinitely differentiable follows from the fact that $\varphi^{\prime}$ is a composition of inverses and continuous bilinear maps (the composition of linear operators).

Remark 2.2.5. By Proposition 2.2.3 the set of invertible linear maps is open, and thus the derivative above really makes sense.

Above we defined the set of invertible linear maps. Let us now treat general maps.

Definition 2.2.6. Let $f \in C(X, Y)$ be a continuous map and let $u \in X$. Then $f$ is said to be locally invertible at $u$ if there exist neighborhoods $U$ of $u$ and $V$ of $f(u)$ and a map $f^{-1}: V \longrightarrow U$ such that

$$
f \circ f^{-1}=I_{V}, \quad f^{-1} \circ f=I_{U} .
$$

We say that $f \in \operatorname{Hom}(U, V)$.

Proposition 2.2.7. The following properties hold:

(i) (Transitivity) Let $f \in C(X, Y)$ be locally invertible at $u \in X$ and $g \in C(Y, Z)$ be locally invertible at $v=f(u)$. Then $g \circ f$ is locally invertible at $u$.

(ii) (Stability) Let $f$ be locally invertible at $u$. Then $f$ is locally invertible at any point in some neighborhood of $u$.

Proof. (i) We have $f \in \operatorname{Hom}\left(U, V^{\prime}\right)$ and $g \in \operatorname{Hom}\left(V^{\prime \prime}, W\right)$. Take $V=V^{\prime} \cap V^{\prime \prime}$. Then

$$
g \circ f: f^{-1}(V) \longrightarrow g(V)
$$

is well defined and is clearly a bijection, thus is an invertible map.

(ii) We have $f \in \operatorname{Hom}(U, V)$. Let $u^{\prime} \in U$ and take some ball $B$ around $u^{\prime}$ contained in $U$. Then $\left.f\right|_{B}: B \longrightarrow f(B)$ is a bijection, thus $f$ is locally invertible at $u^{\prime}$.

Let $f$ be a locally invertible map at $u_{0} \in U$ and suppose that $f$ is differentiable at $u_{0}$ and that $f^{-1}$ is differentiable at $v_{0}=f\left(u_{0}\right)$. We have that

$$
f^{-1} \circ f(u)=u \quad \forall u \in U, \quad f \circ f^{-1}(v)=v \quad \forall v \in V .
$$

Differentiating and applying the chain rule we get

$$
\left(f^{-1}\right)^{\prime}\left(v_{0}\right) \circ f^{\prime}\left(u_{0}\right)=I_{X}, \quad f^{\prime}\left(u_{0}\right) \circ\left(f^{-1}\right)^{\prime}\left(v_{0}\right)=I_{Y} .
$$

Hence, $f^{\prime}\left(u_{0}\right) \in \operatorname{Inv}(X, Y)$ and $\left(f^{\prime}\left(u_{0}\right)\right)^{-1}=\left(f^{-1}\right)^{\prime}\left(v_{0}\right)$.

We have just proved that invertibility of maps implies the invertibility of derivatives. Now, derivatives are linear maps, for which it is much easier to study invertibility. It would 
then be interesting to try and obtain information about the invertibility of maps from the invertibility of derivatives. This is our next theorem.

Theorem 2.2.8 (Inverse Function Theorem). Let $f \in C^{1}(X, Y)$ and suppose $f^{\prime}\left(u_{0}\right) \in$ $\operatorname{Inv}(X, Y)$. Then $f$ is locally invertible at $u_{0}$ with $C^{1}$ inverse. In other words, there exist neighborhoods $U$ of $u_{0}$ and $V$ of $v_{0}=f\left(u_{0}\right)$ such that

(i) $f \in \operatorname{Hom}(U, V)$.

(ii) $f^{-1} \in C^{1}(V, X)$ and

$$
\left(f^{-1}\right)^{\prime}\left(v_{0}\right)=f^{\prime}\left(u_{0}\right)^{-1}
$$

(iii) If $f \in C^{k}(X, Y)$ then $f^{-1} \in C^{k}(V, X)$.

Proof. We begin by making some simplifications to our problem.

If we prove the theorem for $A \circ f$, where $A$ is any invertible linear map, then the theorem is proved for $f$. Indeed, we know that $A^{-1}$ is locally invertible. Then, by the transitivity property, the map

$$
A^{-1} \circ[A \circ f]=f
$$

is locally invertible. Choosing $A=\left[f^{\prime}(0)\right]^{-1}$ we reduce the problem to proving the theorem for $F: X \longrightarrow X, F(u)=\left[f^{\prime}(0)\right]^{-1} \circ f(u)$.

We consider the case where $u_{0}=0$ and $v_{0}=F(0)=0$. The general case follows immediately from this one by considering compositions with translations (which are, of course, locally invertible).

Let $g(u)=u-F(u)$. Note that $g \in C^{1}(X, X)$ and that $g^{\prime}(0)=0$. By continuity, there is some ball of radius $r>0$ such that for $p \in B_{r}(0)$ we have

$$
\left\|g^{\prime}(p)\right\|<\frac{1}{2}
$$

Now, by the Mean Value Inequality (Theorem 2.1.15) we have that

$$
\begin{aligned}
\|g(p)-g(q)\| & \leq \sup _{w \in[p, q]}\left\|g^{\prime}(w)\right\|\|p-q\| \\
& \leq \frac{1}{2}\|p-q\|
\end{aligned}
$$

for all $p, q \in B_{r}(0)$. Hence, $g$ is a contraction on $B_{r}(0)$. In particular,

$$
\|g(p)\| \leq \frac{1}{2}\|p\| \quad \forall p \in B_{r}(0) .
$$

For $v \in X$, define

$$
\phi_{v}(u)=v-g(u) .
$$

Note that $\phi_{v}$ is a contraction in $B_{r}(0)$. Moreover, if for $u \in B_{r}(0)$ and $v \in B_{r / 2}(0)$ we have that

$$
\left\|\phi_{v}(u)\right\| \leq\|v\|+\|g(u)\| \leq r
$$

Then, if we choose $v \in B_{r / 2}(0)$ then $\phi_{v}$ is a contraction that maps $B_{r}(0)$ into itself. Then, by the Banach Fixed Point Theorem it has a unique fixed point $u \in B_{r}(0)$ such that

$$
u=v-g(u)
$$


Thereof,

$$
v=F(u)
$$

Hence $F$ is bijective from $B_{r}(0)$ into $B_{r / 2}(0)$ and thus we can define an inverse

$$
F^{-1}: B_{r / 2}(0) \longrightarrow B_{r}(0)
$$

To show that $F^{-1}$ is continuous let $u=F^{-1}(v)$ and $w=F^{-1}(z)$, that is,

$$
\begin{aligned}
u+g(u) & =v \\
w+g(w) & =z
\end{aligned}
$$

Then

$$
\begin{aligned}
\|u-w\| & \leq\|v-z\|+\|g(u)-g(w)\| \\
& \leq\|v-z\|+\frac{1}{2}\|u-w\|
\end{aligned}
$$

and thus

$$
\left\|F^{-1}(v)-F^{-1}(z)\right\| \leq 2\|v-z\| .
$$

We have thus proved that

$$
\left.F\right|_{U} \in \operatorname{Hom}(U, V)
$$

where $V=B_{r / 2}(0)$ and $U=B_{r}(0) \cap F^{-1}(V)$. Hence (i) is proved.

For (ii), suppose $r$ small enough such that $F^{\prime}(u)$ is invertible. Since $F$ is differentiable at $u$, we may write

$$
F(u)-F(w)=F^{\prime}(u)(u-w)+\|u-w\| \psi(u-w)
$$

where $\psi$ is some function such that

$$
\lim _{w \rightarrow u} \psi(u-w)=0
$$

Now, note that

$$
\begin{aligned}
\left\|F^{-1}(v)-F^{-1}(z)-F^{\prime}(u)^{-1}(v-z)\right\| & =\left\|u-w-F^{\prime}(u)^{-1}(F(u)-F(w))\right\| \\
& =\left\|u-w-u+w+F^{\prime}(u)^{-1}\right\| u-w\|\psi(u-w)\| \\
& \leq C\|v-z\|\left\|\psi\left(F^{-1}(v)-F^{-1}(z)\right)\right\| \\
& \rightarrow 0 .
\end{aligned}
$$

Hence, $F^{-1}$ is differentiable and

$$
\left(F^{-1}\right)^{\prime}(v)=F^{\prime}(u)^{-1}
$$

To see that $\left(F^{-1}\right)^{\prime}: v \mapsto F^{\prime}\left(F^{-1}(v)\right)^{-1}$ is continuous, note that it is a composition of continuous maps:

$$
v \stackrel{F^{-1}}{\rightarrow} u \stackrel{F^{\prime}}{\rightarrow} F^{\prime}(u) \stackrel{\varphi}{\rightarrow}\left(F^{\prime}(u)\right)^{-1} .
$$

Item (iii) follows by induction, using the same arguments presented above and noting that $\varphi \in C^{\infty}$.

Remark 2.2.9. It can be show that the continuity of the derivative cannot be dropped in 
the theorem. Indeed, consider the following elementary counterexample:

$$
f(x)=\left\{\begin{array}{l}
x+2 x^{2} \sin (1 / x), \quad x \neq 0 \\
0, \quad x=0
\end{array}\right.
$$

We have that $f^{\prime}(x)=1+4 x \sin (x)-2 \cos (1 / x)$, so the $\operatorname{limit}_{\lim _{x \rightarrow 0}} f^{\prime}(x)$ does not exist, although $f^{\prime}(0)=1$. Hence it is discontinuous at $x=0$. Moreover, it oscillates up and down, so it is not invertible around the origin.

\subsubsection{The Implicit Function Theorem}

Suppose we know a given point $\left(u_{0}, v_{0}\right)$ is a solution to the equation

$$
f(u, v)=0 .
$$

It may be of interest to find a path of solutions near the given point, that is, to find a map $u \mapsto v(u)$ such that $v\left(u_{0}\right)=v_{0}$ and $f(u, v(u))=0$. In other words, we want to find a function $v=v(u)$ implicitly defined by $f$. But does such a function always exist? If so, what smoothness properties does it have? Does it depend on $f$ ? How so?

The Implicit Function Theorem answers all these questions.

Theorem 2.2.10 (Implicit Function Theorem). Let $U \subset X \times Y$ be an open neighborhood of $\left(u_{0}, v_{0}\right)$ and $f: U \longrightarrow Z$, where $X, Y, Z$ are Banach spaces. Suppose that

$$
f\left(u_{0}, v_{0}\right)=0 .
$$

Also, assume that $f_{v}\left(u_{0}, v_{0}\right)$ exists and is bijective and that both $f$ and $f_{v}$ are continuous at $\left(u_{0}, v_{0}\right)$. Then

(i) There are $r_{0}, r>0$ such that for every $u \in B_{r_{0}}\left(u_{0}\right)$ there is exactly one $v(u) \in B_{r}\left(v_{0}\right) \subset$ $Y$ and $f(u, v(u))=0$.

(ii) The sequence

$$
v_{n+1}=v_{n}-f_{v}^{\prime}\left(u_{0}, v_{0}\right)^{-1} f\left(u, v_{n}(u)\right)
$$

converges to $v(u)$ for $u \in B_{r_{0}}\left(u_{0}\right)$.

(iii) If $f$ is continuous in a neighborhood of $\left(u_{0}, v_{0}\right)$ then $v(\cdot)$ is continuous in a neighborhood of $u_{0}$.

(iv) If $f$ is of class $C^{k}$ then $v(\cdot)$ is of class $C^{k}$.

Proof. Without loss of generality, we can suppose $\left(u_{0}, v_{0}\right)=0$ (it suffices to compose with translations).

Set

$$
g(u, v):=f_{v}^{\prime}(0,0)[v]-f(u, v) .
$$

Then the equation $f(u, v)=0$ is equivalent to

$$
v=f_{v}^{\prime}(0,0)^{-1}[g(u, v)]=: T_{u}(v)
$$

Here $u$ is an index to the function $T$, not a partial derivative. We must show that there exists a unique solution to this equation. 
Let $\|u\|,\|v\|,\|w\| \leq r$. Note that

$$
g_{v}^{\prime}(u, v)=f_{v}^{\prime}(0,0)-f_{v}^{\prime}(u, v)
$$

hence $g_{v}^{\prime}$ is continuous at $(0,0)$ (because $f_{v}^{\prime}$ is) and $g_{v}^{\prime}(0,0)=0$. Then Taylor's Theorem implies that

$$
\|g(u, v)-g(u, w)\| \leq \sup _{0<t<1} \| g_{v}^{\prime}(u, t(v-w)\|\| v-w \| \stackrel{r \rightarrow 0}{\longrightarrow} 0
$$

Moreover, since $g$ is continuous at $(0,0)$ and $g(0,0)=0$,

$$
\|g(u, v)\| \stackrel{r \rightarrow 0}{\longrightarrow} 0
$$

Let $M=\{v \in Y:\|v\| \leq r\}$. Then if $r, r_{0}>0$ are small enough we have that

$$
\left\|T_{u} v\right\| \leq r, \quad\left\|T_{u}(v)-T_{u}(w)\right\| \leq \frac{1}{2}\|v-w\|
$$

for all $v, w \in M$ and fixed $u \in X$ such that $\|u\| \leq r_{0}$.

It then follows that $T_{u}: M \longrightarrow M$ is a contraction. Thus for each fixed $u$ there is a unique fixed point, $v=v(u)$, that is the solution to the equation $f(u, v)=0$. This proves (i).

Item (ii) follows from the construction of the fixed point in the proof of the Banach Fixed Point Theorem.

The continuity of $v$ follows from the continuity of the fixed point on a parameter (see Zeidler (1986), Proposition 1.2). This proves (iii).

It remains to prove (iv). First, let $k=1$. We will compute the Gateaux derivative of $v$ and show that it is continuous. From the existence and continuity of $v(u)$ we have that

$$
f(u+t h, v(u+t h))=f(u, v) .
$$

But since $f$ is differentiable we have

$$
\begin{aligned}
0 & =f(u+t h, v(u+t h))-f(u, v(u)) \\
& =f_{u}^{\prime}(u, v(u))[t h]+f_{v}^{\prime}(u, v(u))[v(u+t h)-v(u)]+o(t) .
\end{aligned}
$$

Then

$$
v(u+t h)-v(u)=-t f_{v}^{\prime}(u, v(u))^{-1} f_{u}^{\prime}(u, v(u))[h]+o(t)
$$

and hence the Gateaux derivative of $v$ exists:

$$
v_{G}^{\prime}(u)[h]=-f_{v}^{\prime}(u, v(u))^{-1} f_{u}^{\prime}(u, v(u))[h]
$$

Note that $f_{v}^{\prime}(u, v(u))$ is invertible in a neighborhood of the origin by Proposition 2.2.3. Since $f$ is of class $C^{1}$ then both $f_{u}^{\prime}$ and $f_{v}^{\prime}$ are continuous, then $v_{G}$ ! is continuous. Thus follows that $v$ is of class $C^{1}$.

Let $k=2$. Note that

$$
G(u)=f_{v}^{\prime}(u, v(u)) v^{\prime}(u)[h]+f_{u}^{\prime}(u, v(u))[h]=0
$$

for all $x$ in a neighborhood of the origin. Therefore $G(u+t k)=0$ for small $t$. Repeating the argument above we can show that the Gateaux derivative of $v$ exists and is continuous. 
For higher $k$, the argument is analogous. The key fact in the argument is the existence of $f_{v}(u, u(v))^{-1}$.

The proof is now complete.

Remark 2.2.11. Note that the proof above gives us an expression for $v^{\prime}(u)$ :

$$
v^{\prime}(u)=-f_{v}^{\prime}(u, v(u))^{-1} f_{u}^{\prime}(u, v(u)) .
$$




\section{Chapter 3}

\section{Genus Theory}

In this chapter we study a topological tool that is of fundamental importance in the proof of our main theorem: the Krasnoselskii genus.

It was first introduced, as the reader may guess, by Krasnoselskii in the study of nonlinear integral equations. The original definition can be found, for example, in Krasnoselskii (1964). We use an equivalent definition by Coffman (1969). The proof of the equivalence can be found in Rabinowitz (1973). For the properties of the genus we follow Kavian (1993). Another interesting reference is Ambrosetti and Malchiodi (2007), which presents some properties of the genus and a general framework for the application of the theory to the existence and multiplicity of critical points of even unbounded functionals.

\subsection{Definitions}

We begin by defining the domain of genus theory.

Definition 3.1.1. Let $X$ be a Banach space. Then $s(X)$ denotes the family of closed, symmetric subsets of $X$ that do not contain the origin:

$$
s(X):=\{A \subset X: A=\bar{A}, A=-A, 0 \notin A\}
$$

We now define the genus of a set in $s(X)$.

Definition 3.1.2. For $A \in s(X)$, the genus of $A$ is the least integer $n$ such that there exist a continuous, odd function between $A$ and $\mathbb{R}^{n} \backslash\{0\}$ :

$$
\gamma(A)=\inf \left\{n \geq 1: \exists \varphi: A \longrightarrow \mathbb{R}^{n} \backslash\{0\} \text { continuous and odd }\right\} .
$$

For convenience, we set $\gamma(\emptyset)=0$. If no such function exists, we set $\gamma(A)=\infty$.

Example 3.1.3. Let $x_{0} \in X$ and $R<\left\|x_{0}\right\|$. Set $A=\overline{B_{R}\left(x_{0}\right)} \cup \overline{B_{R}\left(-x_{0}\right)}$. Of course $A \in s(X)$. Then we can compute $\gamma(A)=1$. Indeed, it suffices to take $\varphi: A \longrightarrow \mathbb{R}$ with $\varphi(x)=1$ if $x \in \overline{B_{R}\left(x_{0}\right)}$ and $\varphi(x)=-1$ otherwise.

We now present an important class of sets of genus $N$.

Example 3.1.4. Let $X=\mathbb{R}^{N}$ and let $\Omega$ be an open, symmetric set containing the origin. Then $\gamma(\partial \Omega)=N$.

It is immediately seen that $\partial \Omega \in s\left(\mathbb{R}^{N}\right)$. Of course we have that $\gamma(\partial \Omega) \leq N$, since the identity is a trivial odd, continuous function from $\partial \Omega$ onto $\mathbb{R}^{N} \backslash\{0\}$. Now, suppose 
$\gamma(\partial \Omega) \leq N-1$. Then there exists and odd, continuous function $\varphi: \partial \Omega \longrightarrow \mathbb{R}^{N-1} \backslash\{0\}$. Then, by the theorem of Borsuk-Ulam (see Kavian (1993), or the Appendix) there is some $x \in \partial \Omega$ such that $\varphi(x)=0$, a contradiction. Hence $\gamma(\partial \Omega)=N$.

\subsection{Properties of the genus}

We now present some of the most useful properties of the genus.

Theorem 3.2.1. Let $X$ be a Banach space and let $A, B \in s(X)$.

(i) If there exist and odd, continuous function $f: A \longrightarrow B$ then $\gamma(A) \leq \gamma(B)$.

(ii) If $A \subset B$ then $\gamma(A) \leq \gamma(B)$.

(iii) If there is and odd homeomorphism $f: A \longrightarrow B$ then $\gamma(A)=\gamma(B)$.

(iv) $\gamma(A \cup B) \leq \gamma(A)+\gamma(B)$.

(v) If $A$ is compact then $\gamma(A)<\infty$.

(vi) If $A$ is compact, it has a closed neighborhood of the same genus. More precisely, there exists an $\varepsilon>0$ such that if $A_{\varepsilon}=\{x \in X: d(x, A) \leq \varepsilon\}$ then $\gamma\left(A_{\varepsilon}\right)=\gamma(A)$.

(vii) If $\gamma(B)<\infty$ then

$$
\gamma(\overline{A \backslash B}) \geq \gamma(A)-\gamma(B)
$$

Proof. (i) If $\gamma(B)=\infty$ there is nothing to show. Assume then that $\gamma(B)=n<\infty$. Then there exists and odd, continuous function $\varphi: B \longrightarrow \mathbb{R}^{n} \backslash\{0\}$. Now, the composition

$$
\varphi \circ f: A \longrightarrow \mathbb{R}^{n} \backslash\{0\}
$$

is an odd, continuous function. Hence $\gamma(A) \leq n$.

(ii) The canonical inclusion is an odd, continuous function from $A$ to $B$, hence the result follows from (i).

(iii) By (i) we have that $\gamma(A) \leq \gamma(B)$ and also the converse inequality, hence $\gamma(A)=\gamma(B)$.

(iv) If the genus of either $A$ or $B$ is $\infty$, then there is nothing to prove. Suppose then that $\gamma(A)=m<\infty, \gamma(B)=n<\infty$. Then there exist two odd, continuous functions

$$
\varphi: A \longrightarrow \mathbb{R}^{m} \backslash\{0\}
$$

and

$$
\psi: B \longrightarrow \mathbb{R}^{n} \backslash\{0\} .
$$

By Tietze's theorem we can extend both functions to odd, continuous functions defined in $X$ :

$$
\tilde{\varphi}: X \longrightarrow \mathbb{R}^{m} \text { odd, continuous such that }\left.\tilde{\varphi}\right|_{A}=\varphi
$$

and

$$
\tilde{\psi}: X \longrightarrow \mathbb{R}^{n} \text { odd, continuous such that }\left.\tilde{\psi}\right|_{B}=\psi \text {. }
$$

Now, set

$$
\begin{aligned}
f: A \cup B & \longrightarrow \mathbb{R}^{m+n} \backslash\{0\} \\
x & \longmapsto(\tilde{\varphi}(x), \tilde{\psi}(x))
\end{aligned}
$$


Note that $f$ is an odd, continuous function and that indeed $f(x) \neq 0$ for all $x \in A \cup B$. Hence $\gamma(A \cup B) \leq m+n$.

(v) Let $x \in A$. Take $R(x)=\|x\| / 2$ and set

$$
\omega(x)=B_{R(x)}(x) \cup B_{R(x)}(-x),
$$

$A(x)=\overline{\omega(x)}$. We know from Example 3.1.3 that $\gamma(A(x))=1$. Now, of course the family $\{\omega(x)\}_{x \in A}$ is an open cover of $A$, hence, by compactness, there is some finite set of points $x_{1}, \ldots, x_{n}$ such that

$$
A \subset \bigcup_{1 \leq i \leq n} \omega\left(x_{i}\right)
$$

and hence

$$
A \subset \bigcup_{1 \leq i \leq n} A\left(x_{i}\right)
$$

By the subaditivity property of the genus ((iv)) we have that $\gamma(A) \leq n$.

(vi) By (v) we know that $\gamma(A)=n<\infty$, so there exists an odd, continuous function $\varphi: A \longrightarrow \mathbb{R}^{n} \backslash\{0\}$. By Tietze's theorem, such a function admits an odd, continuous extension $\tilde{\varphi}: X \longrightarrow \mathbb{R}^{n}$.

By the compactness of $A$, there exists an $\varepsilon>0$ such that $0 \notin \tilde{\varphi}\left(A_{\varepsilon}\right)$. Indeed, suppose there is no such $\varepsilon$. Then there are sequences $\left(x_{k}\right) \subset X,\left(y_{k}\right) \subset A$ such that

$$
\tilde{\varphi}\left(x_{k}\right)=0, \quad d\left(x_{k}, y_{k}\right)=\left\|x_{k}-y_{k}\right\| \leq \frac{1}{k} .
$$

By the compactness of $A$, the sequence $\left(y_{k}\right)$ is convergent (up to a subsequence), $y_{k} \rightarrow y \in A$. Consequently, $x_{k} \rightarrow y$ :

$$
\left\|x_{k}-y\right\| \leq\left\|x_{k}-y_{k}\right\|+\left\|y_{k}-y\right\| \rightarrow 0 \text {. }
$$

But then, since $\varphi$ is continuous, $\tilde{\varphi}(y)=\varphi(y)=0$, a contradiction. Consequently, for some $\varepsilon>0$ we have that $\tilde{\varphi}\left(A_{\varepsilon}\right) \subset \mathbb{R}^{n} \backslash\{0\}$. Hence

$$
n=\gamma(A) \leq \gamma\left(A_{\varepsilon}\right) \leq n .
$$

(vii) Of course we have that $A \subset B \cup \overline{A \backslash B}$. Then

$$
\gamma(A) \leq \gamma(B)+\gamma(\overline{A \backslash B}) .
$$

Since $\gamma(B)<\infty$,

$$
\gamma(\overline{A \backslash B}) \geq \gamma(A)-\gamma(B)
$$

which completes the proof. 


\section{Chapter 4}

\section{Deformations}

The present chapter is devoted to the Deformation Lemma. It basically says that the topological structure of the sublevels of a functional change when we pass through a critical value, or rather that such structure remains the same in a neighborhood of a regular value.

Such a change in topology is useful to characterize the critical levels, and thus provides us with a tool for finding critical points.

We present here a fairly general version, for functionals constrained on a manifold of codimension 1 and such that the map defining the constraint is of class $C_{l o c}^{1,1}$. It can be extended in a number of ways, for example to the case of $C^{1}$ manifolds of any finite codimension, see Bonnet (1993), or to the case of complete $C^{1,1}$-Finsler manifolds, see Palais (1970).

After some motivations to look at the topology of sublevels when dealing with critical points, we present the Palais-Smale condition, the construction of tangent pseudo-gradient vector fields, state and prove the Deformation Lemma and present some of its applications. We also present, briefly, some generalizations that will be useful in Chapter 6 .

Notation. In what follows, let $X$ be a Banach Space and $J: X \longrightarrow \mathbb{R}$ a $C^{1}$ functional. The Fréchet derivative of $J$ is denoted by $J^{\prime}$. Given $b \in \mathbb{R}, J^{b}$ denotes the sublevel $\{x \in X ; J(x) \leq$ $b\}$. The sets $J_{b}$ and so on are defined analogously. $K_{b}:=\left\{u \in X ; J(u)=b, J^{\prime}(u)=0\right\}$ denotes the set of critical points of $J$ at level $b$.

\subsection{Motivation}

The topology of the sublevels of a functional is intrinsically related to the existence of critical points for the given functional.

Example 4.1.1. Consider the real functional $J(x)=x^{3}-3 x$. It is easily seen that $J^{\prime}(x)=$ $3 x^{2}-3$ and so the critical points are $x= \pm 1$, with critical values $c_{1}:=J(1)=-2$ and $c_{2}:=J(1)=2$. Now, note that

- for $a_{1}<c_{1}$ we have $J^{a_{1}}=\left[-\infty, J^{-1}\left(a_{1}\right)\right]$ (one connected component).

- for $c_{1}<a_{2}<c_{2}$ we have $J^{a_{2}}=[-\infty, \alpha] \cup[\beta, \gamma]$ for some $\alpha<\beta<\gamma$ (two connected components).

- for $a_{3}>c_{2}$ we have $J^{a_{3}}=\left[-\infty, J^{-1}\left(a_{3}\right)\right]$ (again, one connected component).

Note that for each $a_{i}$ there is some $\varepsilon>0$ small enough so that $J^{a_{i}}$ and $J^{a_{i}+\varepsilon}$ have the same topological structure, i.e. are homeomorphic, and so can be continuously deformed into one another. For the values $c_{i}$, however, it is impossible to find such $\varepsilon$. Indeed, for small 


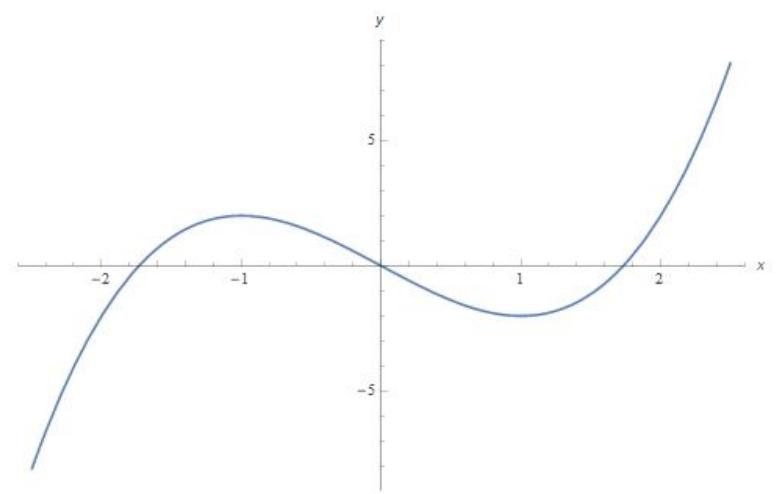

Figure 4.1: Graph of $J(x)=x^{3}-3 x$

values of $\varepsilon>0$ the sets $J^{c_{i}-\varepsilon}$ and $J^{c_{i}+\varepsilon}$ have a different number of connected components and thus are not homeomorphic.

However, a change in the number of connected components is not enough as a criterion for the existence of critical points.

Example 4.1.2. Consider in $\mathbb{R}^{2}$ the functional $J(x, y)=\left(x^{2}+y^{2}\right)^{2}-2\left(x^{2}+y^{2}\right)$.

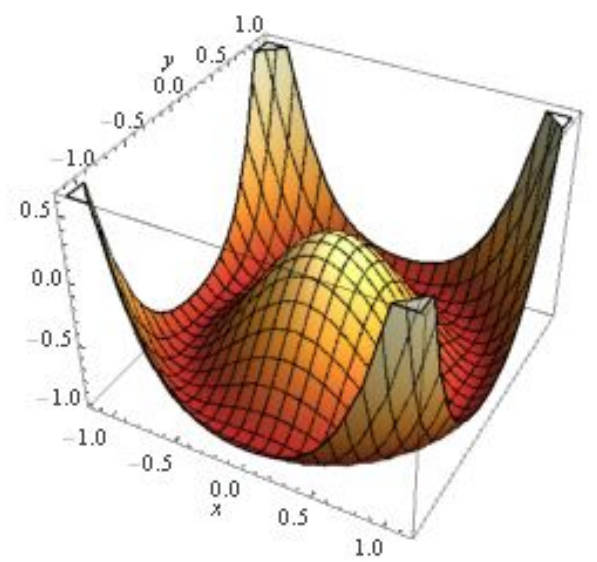

Figure 4.2: Graph of $J(x, y)=\left(x^{2}+y^{2}\right)^{2}-2\left(x^{2}+y^{2}\right)$

It is clear that $J$ has critical values $c_{1}:=-1$ and $c_{2}:=0$. Now, note that

- for $a_{1}<c_{1}$ we have $J^{a_{1}}=\emptyset$

- for $c_{1}<a_{2}<c_{2}$ the sublevel is a ring, with the inner radius shrinking as $a_{2}$ goes to $c_{2}$

- for $a_{3}>c_{2}$ the sublevel is some ball $B_{R}(0)$.

Now, in the last two cases the presence of a critical level did not cause a change in the number of connected sets of the sublevels. Nonetheless, there was a (more subtle) change of topology: while $J^{a_{3}}$ is simply connected, $J^{a_{2}}$ is not.

Now, a change in the topology of sublevels, alone, is not enough to assert the existence of critical points 


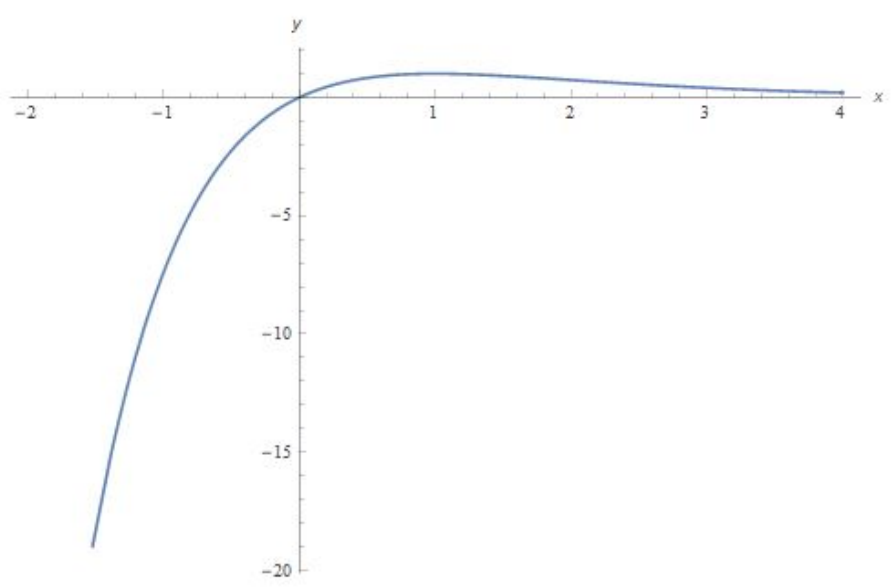

Figure 4.3: Graph of $J(x)=x e^{1-x}$

Example 4.1.3. Consider the real functional $J(x)=x e^{1-x}$ (see Figure 4.3). We have that $J^{\prime}(x)=(1-x) e^{1-x}$, so $J$ has a (maximum) critical point at $x=1$, with critical value $c=1$. We also have that $\lim _{x \rightarrow+\infty} J(x)=0$. Then, although 0 is not a critical level, for any $0<a<c$ the sublevel $J^{a}$ has two connected components, while for $a<0$ the sublevel $J^{a}$ is an interval.

In the previous example, it is intuitive that some compactness is missing. To overcome such an issue, the notion of Palais-Smale condition is introduced.

\subsection{Preliminary definitions}

The formal definition of a constraint is as follows:

Definition 4.2.1. Let $X$ be a Banach space. A constraint is a set

$$
M=\{v \in X ; G(v)=0\}
$$

where $G \in C^{1}(X, \mathbb{R})$ is such that $G^{\prime}(v) \neq 0$ for all $v \in M$.

It can be shown (see Ambrosetti and Malchiodi (2007)) that $M$ is a submanifold of codimension 1 of $X$.

Definition 4.2.2. Let $M$ be a constraint defined by the map $G$ and let $p \in M$. The tangent space to $M$ at the point $p$ is the set

$$
T_{p} M:=\left\{v \in X ; G^{\prime}(p)[v]=0\right\} .
$$

Note that the derivative of the function that defines the restriction is "orthogonal" to the restriction at each point. Indeed, $X=T_{p} M \oplus\left\langle G^{\prime}(p)\right\rangle$.

Definition 4.2.3. Let $J \in C^{1}(X, \mathbb{R})$ be a functional and let $M$ be a constraint defined by $G$. A constrained critical point is a point $u \in M$ such that

$$
J^{\prime}(u)[v]=0 \quad \forall v \in T_{u} M
$$

Equivalently, $u$ is such that

$$
J^{\prime}(u)=\lambda G^{\prime}(u)
$$


The real number $\lambda$ is called Lagrange multiplier.

We provide more details on Lagrange Multipliers in the next chapter.

Note that when dealing with functional restricted to manifolds the critical points are those such that the derivative in the direction of the manifold (that is, in the tangent space) is identically zero.

\subsection{The Palais-Smale condition}

As we have seen in Example 4.1.3, some notion of compactness is necessary if we wish to deal with critical points via topology. The Palais-Smale condition is a notion that emulates the necessary compactness and allows us to construct the tangent pseudo-gradient vector field later on.

Definition 4.3.1. Let $X$ be a Banach space, $J \in C^{1}(X, \mathbb{R})$ and $M$ a constraint defined by the map $G$. Then $\left.J\right|_{M}$ satisfies the Palais-Smale condition if every sequence $\left(u_{n}\right)$ such that

$$
\left(J\left(u_{n}\right)\right) \text { is bounded, }\left.\quad J\right|_{M} ^{\prime}\left(u_{n}\right) \rightarrow 0
$$

has a converging subsequence. If $J\left(u_{n}\right) \rightarrow b$ we say that $J$ satisfies the Palais-Smale condition at level $b$.

Remark 4.3.2. Let $\left(u_{n}\right)$ be a sequence satisfying the Palais-Smale condition. Then there is a sequence $\left(\lambda_{n}\right)$ such that $\lambda_{n} \rightarrow \lambda$, the Lagrange multiplier.

Remark 4.3.3. It is not strictly necessary that the functional be defined in all of $X$. One may ask for a functional defined in a neighborhood of $M$ as well as in $M$ only, if taking care of the definition of the derivative on manifolds.

Remark 4.3.4. With the notation as above, if $\left.J\right|_{M}$ satisfies the Palais-Smale condition then the following sets are compact:

$$
\begin{aligned}
& \left\{(u, \lambda) \in M \times \mathbb{R} ; J(u)=b, J^{\prime}(u)=\lambda G^{\prime}(u)\right\}, \\
& K_{b}:=\left\{u \in M ; J(u)=b \text { and } \exists \lambda \in \mathbb{R} \text { such that } J^{\prime}(u)=\lambda G^{\prime}(u)\right\}, \\
& \cup_{a \leq b \leq c} K_{b}
\end{aligned}
$$

for all $a, c \in \mathbb{R}$.

\subsection{Tangent pseudo-gradient vector fields}

The idea of the proof of the Deformation Lemma in finite dimensions (or in Hilbert spaces in general) is to construct the deformations as flows in the direction of the gradient. Since in general Banach spaces there is no notion of gradient at our disposal, we emulate the necessary properties with the notion of a pseudo-gradient vector field. In the case of constrained functionals, we talk about tangent pseudo-gradient vector fields.

Notation. Let $X$ be a Banach space and let $M$ be a constraint defined by $G$. For $J \in$ $C^{1}(X, \mathbb{R})$ and $u \in M$ denote

$$
\left\|J^{\prime}(u)\right\|_{*}:=\sup \left\{\left|J^{\prime}(u)[y]\right| ; y \in X,\|y\|=1, G^{\prime}(u)[y]=0\right\} .
$$


Note that $\left\|J^{\prime}(u)\right\|_{*}$ is the norm of the projection of $J^{\prime}(u)$ onto the hyperplane tangent to $M$ at $u$. Also note that $\left\|J^{\prime}(u)\right\|_{*}=0$ means precisely that $J^{\prime}(u)=\lambda G^{\prime}(u)$, i.e., $u$ is a critical points of $\left.J\right|_{M}$.

Notation. With the notation as above, denote by $\tilde{M}$ the set of regular points of $\left.J\right|_{M}$, that is,

$$
\tilde{M}:=\left\{u \in M ; \forall \lambda \in \mathbb{R} \quad J^{\prime}(u)-\lambda G^{\prime}(u) \neq 0\right\} .
$$

We now define tangent pseudo-gradient vectors and vector fields.

Definition 4.4.1. Let $X$ be a Banach space, $J \in C^{1}(X, \mathbb{R})$ and $M$ a constraint defined by $G$. Then $v$ is a tangent pseudo-gradient vector of $J$ with respect to $S$ at $u$ if

$$
\left\{\begin{array}{l}
\| v\left(\|\leq 2\| J^{\prime}(u) \|_{*} ;\right. \\
J^{\prime}(u)[v] \geq\left\|J^{\prime}(u)\right\|_{*}^{2} \quad \text { if } \quad G^{\prime}(u)[v]=0 .
\end{array}\right.
$$

A map $V: \tilde{M} \longrightarrow X$ is a tangent pseudo-gradient vector field of $J$ if $V$ is locally Lipschitz in $\tilde{M}$ and $V(u)$ is a tangent pseudo-gradient vector for every $u \in \tilde{M}$.

Remark 4.4.2. Let $u \in S$. It is immediately seen that the the set of tangent pseudogradient vectors at $u$ is convex. It then follows that convex combinations of two or more tangent pseudo-gradient vector fields is also a tangent pseudo-gradient vector field.

Tangent pseudo-gradient vector fields do not always exist, but under some conditions on the constraint we can prove they exist. We require a little more than $C^{1}$ regularity.

Notation. The set of $C^{1}$ functionals such that the derivative is locally Lipschitz from $X$ onto $X^{*}$ is denoted by $C_{l o c}^{1,1}(X, \mathbb{R})$.

Proposition 4.4.3. Let $X$ be a Banach space, $J \in C^{1}(X, \mathbb{R}), G \in C_{\text {loc }}^{1,1}(X, \mathbb{R})$ and $M$ a constraint defined by $G$. Suppose that $J$ is not constant on $M$. Then there exists a map $V$ that is locally Lipschitz in an open neighborhood $N_{\tilde{M}}$ of $\tilde{M}$ and whose restriction to $\tilde{M}$ is a tangent pseudo-gradient vector field. Moreover, if $J$ and $G$ are even then $N_{\tilde{M}}$ can be chosen symmetric with respect to the origin and $V$ is odd.

Proof. Let $u_{0} \in \tilde{M}$. Then, by the definition of $\left\|J\left(u_{0}\right)\right\|_{*}$ there exists $y_{0} \in X$ such that

$$
\left\|y_{0}\right\|=1, \quad G^{\prime}\left(u_{0}\right)\left[y_{0}\right]=0, \quad J^{\prime}\left(u_{0}\right)\left[y_{0}\right] \geq \frac{2}{3}\left\|J^{\prime}\left(u_{0}\right)\right\|_{*} .
$$

Set

$$
v_{0}=\frac{5}{3}\left\|J^{\prime}\left(u_{0}\right)\right\|_{*} y_{0}
$$

Then we have

$$
\left\{\begin{array}{l}
\left\|v_{0}\right\|=\frac{5}{3}\left\|J^{\prime}\left(u_{0}\right)\right\|_{*} \\
J^{\prime}\left(u_{0}\right)\left[v_{0}\right] \geq \frac{10}{9}\left\|J^{\prime}\left(u_{0}\right)\right\|_{*}^{2}, \quad G^{\prime}\left(u_{0}\right)\left[v_{0}\right]=0
\end{array}\right.
$$

Now, since $u_{0} \in M$, then $G^{\prime}\left(u_{0}\right) \neq 0$ and so there exists $z_{0} \in X$ such that $\left\|z_{0}\right\|=1$ and

$$
G^{\prime}\left(u_{0}\right)\left[z_{0}\right] \geq \frac{2}{3}\left\|G^{\prime}\left(u_{0}\right)\right\|
$$

Setting

$$
x_{0}:=\frac{z_{0}}{G^{\prime}\left(u_{0}\right)\left[z_{0}\right]}
$$


we have that

$$
G^{\prime}\left(u_{0}\right)\left[x_{0}\right]=1, \quad\left\|x_{0}\right\|\left\|G^{\prime}\left(u_{0}\right)\right\| \geq \frac{3}{2} .
$$

Now, follows form the continuity of $G^{\prime}$ that there exists $R_{1}>0$ such that for all $u \in B_{R_{1}}\left(u_{0}\right)$ we have

$$
G^{\prime}(u)\left[x_{0}\right] \geq \frac{1}{2}, \quad\left\|x_{0}\right\|\left\|G^{\prime}(u)\right\| \leq 2 .
$$

Then, taking $R<R_{1}$ small enough we have that

$$
v:=v\left(u, u_{0}\right)=v_{0}-\frac{G^{\prime}(u)\left[v_{0}\right]}{G^{\prime}(u)\left[x_{0}\right]} x_{0}
$$

is a tangent pseudo-gradient vector of $J$ at $u$ for all $u \in B_{R}\left(u_{0}\right) \cap \tilde{M}$. Indeed, first note that

$$
\|v\| \leq\left\|v_{0}\right\|+2 \frac{\left|G^{\prime}(u)\left[v_{0}\right]\right|}{G^{\prime}\left(u_{0}\right)\left[z_{0}\right]} .
$$

By continuity, there is $R$ such that for some $0<\alpha<2$ we have

$$
\left\|v_{0}\right\| \leq \alpha\left\|J^{\prime}(u)\right\|_{*}
$$

and

$$
\left|G^{\prime}(u)\left[v_{0}\right]\right| \leq \frac{2-\alpha}{2} G^{\prime}\left(u_{0}\right)\left[z_{0}\right]\left\|J^{\prime}(u)\right\|_{*}
$$

from what follows that

$$
\|v\| \leq 2\left\|J^{\prime}(u)\right\|_{*}
$$

Now, note that we can find an $R>0$ small enough such that for all $u \in B_{R}\left(u_{0}\right) \cap \tilde{M}$ we have

$$
\begin{aligned}
J(u)[v] & =J^{\prime}(u)\left[v_{0}\right]-\frac{G^{\prime}(u)\left[v_{0}\right]}{G^{\prime}(u)\left[x_{0}\right]} J^{\prime}(u)\left[x_{0}\right] \\
& =J^{\prime}(u)\left[v_{0}\right]-\frac{G^{\prime}(u)\left[v_{0}\right]}{G^{\prime}(u)\left[z_{0}\right]} J^{\prime}(u)\left[z_{0}\right] \\
& \geq\left(\frac{10}{9}-\varepsilon\right)\left\|J^{\prime}(u)\right\|_{*}^{2}-\frac{\left|G^{\prime}(u)\left[v_{0}\right]\right|}{\left|G^{\prime}(u)\left[z_{0}\right]\right|}\left\|J^{\prime}(u)\right\|_{*} \\
& \geq\left(\frac{10}{9}-\varepsilon\right)\left\|J^{\prime}(u)\right\|_{*}^{2}-\left(\frac{3}{2}\left\|G^{\prime}\left(u_{0}\right)\right\|+\varepsilon^{\prime}\right) \mid G^{\prime}(u)\left[v_{0}\right]\|\| J^{\prime}(u) \|_{*} \\
& \geq\left(\frac{10}{9}-\varepsilon\right)\left\|J^{\prime}(u)\right\|_{*}^{2}-\left(\frac{3}{2}\left\|G^{\prime}\left(u_{0}\right)\right\|+\varepsilon^{\prime}\right) \mid G^{\prime}(u)\left[v_{0}\right] \sup _{B_{R}\left(u_{0}\right)}\left\|J^{\prime}(u)\right\|_{*} \\
& \geq\left\|J^{\prime}(u)\right\|_{*}^{2}
\end{aligned}
$$

Various $R$ where considered, it suffices to take the smallest one.

Let us show now that

$$
u \mapsto v\left(u, u_{0}\right)
$$

is a locally Lipschitz map in $B_{R}\left(u_{0}\right)$. Let $K$ be some compact subset. Then, since $G^{\prime}$ is locally 
Lipschitz, for $u, w \in K$ we have:

$$
\begin{aligned}
\left\|v\left(u, u_{0}\right)-v\left(w, u_{0}\right)\right\| & =\left\|\frac{G^{\prime}(u)\left[v_{0}\right]}{G^{\prime}(u)\left[x_{0}\right]} x_{0}-\frac{G^{\prime}(w)\left[v_{0}\right]}{G^{\prime}(w)\left[x_{0}\right]} x_{0}\right\| \\
& \leq \frac{1}{\left|G^{\prime}\left(u_{0}\right)\left[z_{0}\right]\right|}\left\|\frac{G^{\prime}(u)\left[v_{0}\right]}{G^{\prime}(u)\left[x_{0}\right]}-\frac{G^{\prime}(w)\left[v_{0}\right]}{G^{\prime}(w)\left[x_{0}\right]}\right\| \\
& =c\left\|\frac{G^{\prime}(u)\left[v_{0}\right]}{G^{\prime}(u)\left[x_{0}\right]}-\frac{G^{\prime}(w)\left[v_{0}\right]}{G^{\prime}(u)\left[x_{0}\right]}-\left(\frac{G^{\prime}(w)\left[v_{0}\right]}{G^{\prime}(w)\left[x_{0}\right]}-\frac{G^{\prime}(w)\left[v_{0}\right]}{G^{\prime}(u)\left[x_{0}\right]}\right)\right\| \\
& \leq c\left\|G^{\prime}(u)-G^{\prime}(w)\right\|\left\|v_{0}\right\|+\left\|\frac{G^{\prime}(w)\left[v_{0}\right]}{G^{\prime}(w)\left[x_{0}\right]}-\frac{G^{\prime}(w)\left[v_{0}\right]}{G^{\prime}(u)\left[x_{0}\right]}\right\| \\
& =c\|u-w\|+\left\|\frac{G^{\prime}(w)\left[v_{0}\right]}{G^{\prime}(w)\left[x_{0}\right]}-\frac{G^{\prime}(w)\left[v_{0}\right]}{G^{\prime}(u)\left[x_{0}\right]}\right\|
\end{aligned}
$$

Now,

$$
\begin{aligned}
\left\|\frac{G^{\prime}(w)\left[v_{0}\right]}{G^{\prime}(w)\left[x_{0}\right]}-\frac{G^{\prime}(w)\left[v_{0}\right]}{G^{\prime}(u)\left[x_{0}\right]}\right\| & \leq \sup _{K}\left|G^{\prime}(w)\left[v_{0}\right]\right|\left\|\frac{1}{G^{\prime}(w)\left[x_{0}\right]}-\frac{1}{G^{\prime}(u)\left[x_{0}\right]}\right\| \\
& \leq 4 \sup _{K}\left|G^{\prime}(w)\left[v_{0}\right]\right|\left\|G^{\prime}(u)\left[x_{0}\right]-G^{\prime}(w)\left[x_{0}\right]\right\| \\
& \leq c\|u-v\|
\end{aligned}
$$

and thus $u \mapsto v\left(u, u_{0}\right)$ is locally Lipschitz.

Since

$$
N_{\tilde{M}}:=\bigcup_{\tilde{M}} B_{R}(u)
$$

is paracompact (because it is a subset of a metric space) there is a locally finite refinement $\left(\omega_{j}\right)_{j \in I}$ and a partition of unity $\left(\theta_{j}\right)_{j \in I}$ subordinated to $\left(\omega_{j}\right)_{j \in I}$. For each $j \in I$ there is some $u_{0 j}$ such that $\omega_{j} \subset B_{R}\left(u_{0 j}\right)$. Then

$$
V(u)=\sum_{j}=\theta_{j}(u) v\left(u, u_{0 j}\right)
$$

is a locally Lipschitz tangent pseudo-gradient vector field for $J$ over $\tilde{M}$.

Suppose now that $G$ and $J$ are even. Then, taking $R(u)$ as $\min \{R(u), R(-u)\}$ we have that

$$
\bigcup_{\tilde{M}} B_{R}(u)
$$

is symmetric with respect to the origin. Then

$$
V_{1}(u)=\frac{1}{2}(V(u)-V(-u))
$$

is an odd tangent pseudo-gradient vector field. It suffices to show that $V_{-}:=-V(-u)$ is a tangent pseudo-gradient vector for $J$ at $u \in \tilde{M}$. Note that, by the symmetry of $G$ and $J$ the construction of $y_{0}, z_{0}$ and $x_{0}$ is the same for both $u_{0}$ and $-u_{0}$. Thus, if $u \in B_{R}\left(u_{0}\right)$ and $-u \in B_{R}\left(-u_{0}\right)$

$$
v\left(u, u_{0}\right)=v\left(-u,-u_{0}\right) .
$$

It then follows that $V(-u)$ is a convex combination of tangent pseudo-gradient vectors at 
$u$, and thus the claim follows.

Remark 4.4.4. Note that for all $u \in B_{R}\left(u_{0}\right)$ we have

$$
G^{\prime}(u)[v]=G^{\prime}(u)\left[v_{0}\right]-\frac{G^{\prime}(u)\left[v_{0}\right]}{G^{\prime}(u)\left[x_{0}\right]} G^{\prime}(u)\left[x_{0}\right]=0 .
$$

This implies that $G^{\prime}(u)[V(u)]=0$ for all $u \in N_{\tilde{M}}$.

\subsection{The Deformation Lemma and its consequences}

We are now in position to prove the Deformation Lemma for constrained functionals. We remark that it would suffice to consider a functional defined in a neighborhood of the manifold $M$.

Theorem 4.5.1 (Deformation Lemma). Let $X$ be a Banach space, $G \in C_{\text {loc }}^{1,1}(X, \mathbb{R})$ defining the constraint $M, J \in C^{1}(X, \mathbb{R})$ such that $\left.J\right|_{M}$ satisfies the Palais-Smale condition, $\left.J\right|_{M}$ is not constant and $b \in \mathbb{R}$ is not a critical value for $\left.J\right|_{M}$. Then there exists $\varepsilon_{0}>0$ such that for all $0<\varepsilon<\varepsilon_{0}$ there exists a map $\eta \in C(\mathbb{R} \times M, M)$ such that

(i) $\eta(0, u)=u$ for all $u \in M$;

(ii) If $\left.u \notin J\right|_{M_{b-\varepsilon_{0}}^{b+\varepsilon_{0}}}$ then $\eta(t, u)=u$ for all $t \in \mathbb{R}$;

(iii) For each $t \in \mathbb{R} \eta(t, \cdot)$ is a homeomorphism from $M$ onto $M$;

(iv) For all $u \in M$ the function $\left.t \mapsto J\right|_{M}(\eta(t, u))$ is decreasing;

(v) $\left.\eta\left(1,\left.J\right|_{M} ^{b+\varepsilon}\right) \subset J\right|_{M} ^{b-\varepsilon}$;

(vi) If $\left.J\right|_{M}$ and $G$ are even then $\eta(t, \cdot)$ odd.

Proof. The idea is to build a flow as the solution of a convenient ordinary differential equation and then to check that it verifies conditions (i) to (vi).

We know by Proposition 4.4.3 that there exists a map $V$ defined in an open neighborhood $N_{\tilde{M}}$ of $\tilde{M}$, locally Lipschitz over $N_{\tilde{M}}$ such that $\left.V\right|_{\tilde{M}}$ is a tangent pseudo-gradient vector field. If $\left.J\right|_{M}$ and $G$ are even then $N_{\tilde{M}}$ is symmetric with respect to the origin.

Let us note that there exists $\varepsilon_{1}>0$ and $\delta>0$ such that

$$
\left\|\left.J\right|_{M} ^{\prime}(u)\right\|_{*} \geq\left.\delta \quad \forall u \in J\right|_{M b-\varepsilon_{1}} ^{b+\varepsilon_{1}}
$$

It is convenient to choose $\delta \leq 1$. Indeed, suppose that the assertion is not true. Then there are sequences $\varepsilon_{n} \rightarrow 0, \delta_{n} \rightarrow 0$ such that for all $n \in \mathbb{N}$

$$
\left.\exists u_{n} \in J\right|_{M b-\varepsilon_{n}} ^{b-\varepsilon_{n}} \quad \text { such that } \quad\left\|\left.J\right|_{M} ^{\prime}\left(u_{n}\right)\right\|<\delta_{n} .
$$

But then $\left(u_{n}\right)$ is a Palais-Smale sequence at level $b$ for $J$. Hence there is a converging subsequence, which implies, by continuity, that $b$ is a critical level, a contradiction.

Define

$$
\varepsilon_{0}:=\min \left\{\varepsilon_{1}, \frac{\delta^{2}}{8}\right\} .
$$


For each $v \in \tilde{M}$ set $R_{v}=\frac{1}{2} d\left(v, X \backslash N_{\tilde{M}}\right)$. Define

$$
\Omega:=\bigcup_{\tilde{M}} B_{R_{v}}(v)
$$

Now, for $0<\varepsilon<\varepsilon_{0}$ define

$$
A:=\left.\left.J\right|_{M} ^{b-\varepsilon_{0}} \cup J\right|_{M b+\varepsilon_{0}} \cup(X \backslash \Omega), \quad B:=\left.J\right|_{M b-\varepsilon} ^{b+\varepsilon}
$$

and

$$
\alpha(u)=\frac{d(u, A)}{d(u, A)+d(u, B)} .
$$

Of course, $\alpha(B)=\{1\}$ and $\alpha(A)=\{0\}$. We also know that $\alpha$ is locally Lipschitz in $X$. In case $\left.J\right|_{M}$ and $G$ are even then $A$ and $B$ are symmetric with respect to the origin and $\alpha$ is even.

Let

$$
W(u):=\left\{\begin{array}{l}
\alpha(u) \min \left\{1, \frac{1}{\|V(u)\|}\right\} V(u), \quad u \notin A \\
0 \quad \text { otherwise }
\end{array}\right.
$$

Then $W$ is locally Lipschitz and $\|W(u)\| \leq 1$ for all $u \in X$. It then follows that given $u \in X$ there exists a unique solution to the problem

$$
(*)\left\{\begin{array}{l}
\frac{\mathrm{d}}{\mathrm{d} t} \eta(t, u)=-W(\eta(t, u)) \\
\eta(0, u)=u
\end{array}\right.
$$

defined on the whole real line. We know that $\eta(\cdot, u) \in C^{1}(\mathbb{R}, X)$, that $\eta(t, \eta(s, u))=$ $\eta(t+s, u)$ and that $\eta(t, \cdot)$ is an homeomorphism of $X$ onto $X$ for all $t \in \mathbb{R}$. It remains to prove that $\eta$ satisfies properties (i) to (vi).

Property (i) is trivial, by the initial condition of the problem $(*)$.

Property (ii) is trivial, since for $\left.u \notin J\right|_{M b-\varepsilon_{0}} ^{b+\varepsilon_{0}}$ the right hand side of the equation in $(*)$ is zero and thus the solution is constant.

To prove property (iii) it suffices to show that if $u \in M$ then $\eta(t, u) \in M$ for all $t \in \mathbb{R}$. Indeed, the inverse is given by $\eta(-t, u)$ and continuity of both follows from continuity of the solution to $(*)$ with respect to initial conditions. The claim is trivial if $\left.u \notin J\right|_{M b-\varepsilon_{0}} ^{b+\varepsilon_{0}}$. Let $\left.u \in J\right|_{M b-\varepsilon_{0}} ^{b+\varepsilon_{0}}$. Then, $u \in \tilde{M}$. Since $t \mapsto \eta(t, u)$ is continuous we have that $\eta(t, u) \in N_{\tilde{M}}$ for $t$ small enough. But then we have that

$$
\begin{aligned}
\frac{\mathrm{d}}{\mathrm{d} t} G(\eta(t, u)) & =G^{\prime}(\eta(t, u))\left[\frac{\mathrm{d}}{\mathrm{d} t} \eta(t, u)\right] \\
& =-\alpha(\eta(t, u)) \min \left\{1, \frac{1}{\|V(\eta(t, u))\|}\right\} G^{\prime}(\eta(t, u))[V(\eta(t, u))] \\
& =0 .
\end{aligned}
$$

Thus $t \mapsto G(\eta(t, u))$ is constant, which implies that $\eta(t, u) \in M$ for all $t \in \mathbb{R}$. 
To prove (iv), note that

$$
\begin{aligned}
\left.\frac{\mathrm{d}}{\mathrm{d} t} J\right|_{M}(\eta(t, u)) & =\left.J\right|_{M} ^{\prime}(\eta(t, u))\left[\frac{\mathrm{d}}{\mathrm{d} t} \eta(t, u)\right] \\
& =-\left.\alpha(\eta(t, u)) \min \left\{1, \frac{1}{\|V(\eta(t, u))\|}\right\} J\right|_{M} ^{\prime}(\eta(t, u))[V(\eta(t, u))] \\
& \leq-\alpha(\eta(t, u)) \min \left\{1, \frac{1}{\|V(\eta(t, u))\|}\right\}\left\|\left.J\right|_{M} ^{\prime}(\eta(t, u))\right\|_{*}^{2} \\
& \leq 0 .
\end{aligned}
$$

For (v), since we have (iv) it suffices to prove that $\left.J\right|_{M}(\eta(1, u)) \leq b-\varepsilon$ for $\left.u \in J\right|_{M_{b-\varepsilon}^{b+\varepsilon}} ^{b}$. First, note that

$$
\left\|\left.J\right|_{M} ^{\prime}(u)\right\|_{*}^{2} \leq\left. J\right|_{M} ^{\prime}(u)[V(u)] \leq\left\|\left.J\right|_{M} ^{\prime}(u)\right\|_{*}\|V(u)\|,
$$

thus $\left\|\left.J\right|_{M} ^{\prime}(u)\right\|_{*} \leq\|V(u)\| \leq\left. 2|| J\right|_{M} ^{\prime}(u) \|_{*}$. Then we have that

$$
\begin{aligned}
\left.\frac{\mathrm{d}}{\mathrm{d} t} J\right|_{M}(\eta(t, u)) & \leq-\alpha(\eta(t, u)) \min \left\{1, \frac{1}{\|V(\eta(t, u))\|}\right\}\left\|\left.J\right|_{M} ^{\prime}(\eta(t, u))\right\|_{*}^{2} \\
& \leq-\frac{1}{4} \min \left\{1, \frac{1}{\|V(\eta(t, u))\|}\right\}\|V(\eta(t, u))\|^{2} \\
& \leq \begin{cases}-\frac{1}{4} & \text { if }\|V(\eta(t, u))\| \geq 1 \\
-\frac{\delta^{2}}{4} & \text { if }\|V(\eta(t, u))\|<1\end{cases}
\end{aligned}
$$

Integrating, we conclude that

$$
\left.J\right|_{M}(\eta(1, u)) \leq-\frac{\delta^{2}}{4}+\left.J\right|_{M}(\eta(0, u)) \leq-\frac{\delta^{2}}{4}+b+\varepsilon \leq b-\varepsilon
$$

At last, note that if $\left.J\right|_{M}$ and $G$ are even, then $V$, and consequently $W$, can be taken to be odd. In this case, it is easy to see that $-\eta(t, u)$ is a solution to the Cauchy problem $(*)$ with initial condition $-\eta(0, u)=-u$. By the uniqueness of the solution, $\eta(t, \cdot)$ is odd for all $t \in \mathbb{R}$.

Remark 4.5.2. There are plenty versions of the Deformation Lemma. One of them does not require that $b$ be a regular value for $\left.J\right|_{M}$. In this case, the deformation is takes the complement of a neighborhood of the critical points to a lower sublevel. The proof is very similar, and the key ideas are the same. See Struwe (2008) for details.

We now analyze some important consequences of the Deformation Lemma.

Theorem 4.5.3 (Morse Theorem). Let $X$ be a Banach space, $M$ be a constraint defined by $G \in C_{\text {loc }}^{1,1}(X, \mathbb{R}), J \in C^{1}(X, \mathbb{R})$ such that $\left.J\right|_{M}$ is not constant and $b$ a regular value. Then there exists $\varepsilon_{0}>0$ such that for all $0<\varepsilon<\varepsilon_{0}$ the sublevel $\left.J\right|_{M} ^{b-\varepsilon}$ is a deformation retract of $\left.J\right|_{M} ^{b+\varepsilon}$.

Proof. With the notation as in the proof of Theorem 4.5.1, define

$$
\varphi(t, u)=\eta\left(\frac{4 t}{\delta^{2}}\left(\left.J\right|_{M}(u)-b+\varepsilon\right)^{+}, u\right)
$$


We show that $\varphi$ defines a deformation of $J^{b+\varepsilon}$ into $J^{b-\varepsilon}$. That is, $\varphi \in C\left([0,1] \times\left. J\right|_{M} ^{b+\varepsilon},\left.J\right|_{M} ^{b-\varepsilon}\right)$ is such that

$$
\varphi(0, \cdot)=\mathrm{Id}, \quad \varphi(t, \cdot)=\mathrm{Id} \quad \forall t \in[0,1], \quad \varphi\left(1,\left.J\right|_{M} ^{c+\varepsilon}\right)=\left.J\right|_{M} ^{c-\varepsilon} .
$$

Of course, $\varphi(0, \cdot)=\eta(0, \cdot)=$ Id. If $\left.u \in J\right|_{M} ^{b-\varepsilon}$ then $\left.J\right|_{M}(u)-b+\varepsilon \leq-2 \varepsilon<0$ so $\varphi(t, u)=\eta(0, u)=u$ as well.

Now, let $\left.u \in J\right|_{M_{b-\varepsilon}^{b+\varepsilon}} ^{b+a n d} \tau>0$ such that $\left.\eta(t, u) \in J\right|_{M_{b-\varepsilon}^{b+\varepsilon}} ^{b}$ for $0 \leq t \leq \tau$. Recall from the proof of Theorem 4.5.1 that

$$
b-\varepsilon<\left.J\right|_{M}(\eta(\tau, u)) \leq\left. J\right|_{M}(u)-\frac{\delta^{2}}{4} \tau
$$

that is,

$$
\tau<\frac{4}{\delta^{2}}\left(\left.J\right|_{M}(u)-b+\varepsilon\right)=: t_{0}
$$

Then

$$
\varphi(1, u)=\left.\eta\left(t_{0}, u\right) \in J\right|_{M} ^{b-\varepsilon}
$$

Indeed, note that

$$
\left.J\right|_{M}\left(\eta\left(t_{0}, u\right)\right) \leq\left. J\right|_{M}(u)-\frac{\delta^{2}}{4} t_{0}=b-\varepsilon,
$$

which completes the proof.

Theorem 4.5.4 (Minimax Principle). Let $X$ be a Banach space, $M$ be a constraint defined by $G \in C_{\text {loc }}^{1,1}(X, \mathbb{R}), J \in C^{1}(X, \mathbb{R})$ such that $\left.J\right|_{M}$ is not constant and satisfies the PalaisSmale condition, and let $\mathcal{B}$ a nonempty family of nonempty subsets of $M$. Suppose that for each $b \in \mathbb{R}$ and $\varepsilon>0$ the flow $\eta$ constructed in the proof of the Deformation Lemma is such that

$$
\eta(1, A) \in \mathcal{B} \quad \forall A \in \mathcal{B}
$$

Let

$$
\tilde{b}:=\left.\inf _{A \in \mathcal{B}} \sup _{v \in A} J\right|_{M}(v) .
$$

Then if $\tilde{b} \in \mathbb{R}$ we have that $\tilde{b}$ is a critical value of $\left.J\right|_{M}$.

Proof. Suppose $\tilde{b} \in \mathbb{R}$ and is not a critical point of $\left.J\right|_{M}$. By the definition of $\tilde{b}$, there is an $\varepsilon>0$ small enough such that for some $A \in \mathcal{B}$ we have

$$
\tilde{c} \leq\left.\sup _{v \in A} J\right|_{M}(v) \leq \tilde{b}+\varepsilon .
$$

But then $\left.\eta(1, A) \in \mathcal{B} \subset J\right|_{M} ^{\tilde{b}-\varepsilon}$, a contradiction.

The families $\mathcal{B}$ of sets used in the Minimax Principle are defined with the help of the Ljusternik-Schnirelman category (see Ambrosetti and Malchiodi (2007)) or of the genus. For example, we have the following theorem:

Theorem 4.5.5. Let $X$ be a Banach space, $G \in C_{\text {loc }}^{1,1}(X, \mathbb{R}), M$ a constraint defined by $G$ and $J \in C^{1}(X, \mathbb{R})$. Suppose that $G$ and $\left.J\right|_{M}$ are even, that $\left.J\right|_{M}$ is not constant and satisfies the Palais-Smale condition on $M$ and that $0 \notin M$. For $k \in \mathbb{N}$, set

$$
\mathcal{B}_{k}=\{A \in s(X): A \subset M, \gamma(A) \geq k\}
$$


and

$$
b_{k}:=\left.\inf _{A \in \mathcal{B}_{k}} \sup _{v \in A} J\right|_{M}(v) .
$$

Then

(i) For all $k \in \mathbb{N}$ such that $\mathcal{B}_{k} \neq \emptyset$ and $b_{k} \in \mathbb{R}, b_{k}$ is a critical value of $\left.J\right|_{M}$. Moreover,

$$
b_{k} \leq b_{k+1}
$$

and if for some $j \in \mathbb{N}$ we have $\mathcal{B}_{j+k} \neq \emptyset$ and $b_{k}=b_{j+k} \in \mathbb{R}$ then $\gamma\left(K_{b_{k}}\right) \geq j+1$.

(ii) If for all $k \in \mathbb{N}$ we have $\mathcal{B}_{k} \neq \emptyset$ and $b_{k} \in \mathbb{R}$, then

$$
\lim _{n \rightarrow \infty} b_{k}=+\infty
$$

\subsection{Generalizations}

As already mentioned, the theorems of this chapter can be modified to more general situations.

Of remarkable interest is the following theorem, which gives a Minimax Principle for manifolds that are only $C^{1}$ :

Theorem 4.6.1. Let $M$ be a closed symmetric submanifold of a Banach space $X$ such that $0 \notin M$. Let $J \in C^{1}(M, \mathbb{R})$ be even and bounded from below. Define

$$
b_{k}=\inf _{A \in \mathcal{B}_{k}} \sup _{v \in A} J(v)
$$

where $\mathcal{B}_{k}=\{A \in s(X): A \subset M, \gamma(A) \geq k, A$ is compact $\}$. If $\mathcal{B}_{k} \neq \emptyset$ for some $k$ and if $J$ satisfies the Palais-Smale condition then $J$ has at least $k$ distinct pairs of critical points.

The proof relies heavily on the category of Ljusternik-Schnirelman and on the Finsler structure of the manifold. See Szulkin (1988).

There is also a generalization of the category and of the genus and for the results presented here to variational problems that admit some kind of index theory. See Struwe (2008) and the references therein. 


\section{Chapter 5}

\section{Submersions, Manifolds, Lagrange Multipliers}

In the proof of Theorem 1.0.1, we will consider the restriction of a functional to a subset of our Hilbert space. The fact that this subset is a manifold has a fundamental role in the theorems we will apply.

In this chapter, we recall some definitions and prove two important theorems: Ljusternik's theorem on the construction of manifolds via submersions and the Theorem of Lagrange Multipliers.

\subsection{Submersions and submanifolds}

Definition 5.1.1. Let $X$ be a Banach space. A subset $A$ of $X$ is a (smooth) submanifold of $X$ if for every $u \in A$ there exists a neighborhood $U_{u}$ of $u$ in $X$ and a (smooth) map $\xi_{u}: U_{u} \longrightarrow V_{u}$, where $V_{u}$ is an open subset of $X$, and a closed complemented subspace $S$ of $X$ such that

$$
\xi_{u}\left(U_{u} \cap A\right)=V_{u} \cap S .
$$

Definition 5.1.2. Let $X, Y$ be two Banach spaces. A map $G: X \longrightarrow Y$ is said to be a submersion at $u_{0} \in X$ if

(i) $G$ is continuously differentiable in a neighborhood of $u_{0}$;

(ii) $G^{\prime}\left(u_{0}\right)$ is surjective;

(iii) there exists a continuous projection operator $P: X \longrightarrow \operatorname{ker} G^{\prime}\left(u_{0}\right)$. Hence,

$$
X=\operatorname{ker} G^{\prime}\left(u_{0}\right) \oplus(I-P)(X) .
$$

Remark 5.1.3. If $X$ is a Hilbert space and $Y=\mathbb{R}^{k}$ then condition (iii) is immediately satisfied and the map $G=\left(G_{1}, \ldots, G_{k}\right)$ is a submersion at $u_{0}$ if and only if $G_{1}^{\prime}\left(u_{0}\right), \ldots, G_{k}^{\prime}\left(u_{0}\right)$ are linearly independent.

Definition 5.1.4. Let $M \subset X$ and $u_{0} \in M$ be fixed.

(i) An admissible curve in $M$ through $u_{0}$ is a map $u: t \in(-\varepsilon, \varepsilon) \mapsto u(t) \in M$ such that $u(0)=u_{0}$ and the derivative $u^{\prime}(0)$ exists.

(ii) $h$ is called a tangent vector to $M$ at $u_{0}$ if and only if there is an admissible curve such that $h=u^{\prime}(0)$. 
(iii) If the set of tangent vectors form a vector space then this space is denoted by $T_{u_{0}} M$ and called the tangent space at $u_{0}$.

We have the following theorem for the construction of manifolds by means of submersions:

Theorem 5.1.5 (Ljusternik). Let $X, Y$ be Banach spaces and let $G: X \longrightarrow Y$ be a submersion at $u_{0}$ with $G\left(u_{0}\right)=0$. Let $M=G^{-1}(0)$. Then

(i) $T_{u_{0}} M=\operatorname{ker} G^{\prime}\left(u_{0}\right)$.

(ii) There exists a homeomorphism $\varphi: V \subset T_{u_{0}} M \longrightarrow M$ of a neighborhood of 0 onto a neighborhood of $u_{0}$ in $M$. Moreover, $\varphi$ is differentiable and $\varphi^{\prime}(0)=I$.

(iii) If the domain $D(G)$ of $G$ is open and $G$ is a submersion for all $u \in M$ then $M$ is a $C^{1}$-manifold. If $G$ is of class $C^{k}$ them $M$ is a $C^{k}$-manifold.

Proof. The proof relies heavily on the Implicit Function Theorem.

Let

$$
X=X_{1} \oplus X_{2}
$$

where $X_{1}=\operatorname{ker} G^{\prime}\left(u_{0}\right)=P X$ and $X_{2}=(I-P) X$. Thus

$$
u=u_{1}+u_{2}, \quad u_{1} \in X_{1}, u_{2} \in X_{2} .
$$

We assume $X_{1} \neq\{0\}$.

We want to solve the equation

$$
F\left(u_{1}, u_{2}\right)=G\left(u_{0}+u_{1}+u_{2}\right)=0
$$

in terms of a function $u_{2}=\psi\left(u_{1}\right)$, as given by the Implicit Function Theorem, in a neighborhood of the origin in $X_{1}$. Let us verify that the hypothesis of the theorem are satisfied. Suppose $F$ defined on a neighborhood $U$ of $(0,0)$.

First, note that $F(0,0)=G\left(u_{0}\right)=0$.

According to the Chain Rule, we have

$$
F_{u_{i}}\left(u_{1}, u_{2}\right)[h]=G^{\prime}\left(u_{0}+u_{1}+u_{2}\right)[h], \quad h \in X_{i}
$$

on $U$. So both partial derivatives exist and are continuous, thus $F \in C^{1}(U)$.

Now, note that $F_{u_{2}}(0,0)$ is the restriction of $G^{\prime}\left(u_{0}\right)$ to $X_{2}$. It is a continuous linear transformation, as a derivative. Also, it is bijective. Indeed, by hypothesis the range of $G^{\prime}\left(u_{0}\right)$ is $Y$, since it is a submersion. On the other hand, $h \in X_{2}$ and $G^{\prime}\left(u_{0}\right)[h]=0$ if and only if $h=0$, by the way we defined $X_{2}$.

By the Implicit Function Theorem there are $\delta>0$ and a neighborhood $W$ of $(0,0)$ in $X_{1} \times X_{2}$ such that for each $u_{1} \in B_{\delta}(0) \subset X_{1}$ there is one $\psi\left(u_{1}\right) \in X_{2}$ such that $\left(u_{1}, \psi\left(u_{1}\right)\right) \in$ $W$ and

$$
F\left(u_{1}, \psi\left(u_{1}\right)\right)=0
$$

and $\psi \in C^{1}$. Differentiating with respect to $u_{1}$ we have

$$
F_{u_{1}}(0,0)+F_{u_{2}}(0,0) \psi^{\prime}(0)=0 \text {. }
$$

But $F_{u_{1}}(0,0)=\left.G^{\prime}\left(u_{0}\right)\right|_{X_{1}}=0$, thus $\psi^{\prime}(0)=0=\psi(0)$. Then

$$
\psi\left(u_{1}\right)=o\left(u_{1}\right) .
$$


Now set

$$
\varphi\left(u_{1}\right):=u_{0}+u_{1}+\psi\left(u_{1}\right)
$$

This is an homeomorphism of a sufficiently small neighborhood of zero in $X_{1}$ onto a neighborhood of $u_{0}$ in $M$. Indeed:

- Injectivity follows from the injectivity of $\psi$.

- For each $u$ in a neighborhood of $u_{0}$ we have $u=\varphi(P[u])$, thus $\varphi$ is surjective.

- Continuity follows from $\psi\left(u_{1}\right)=o\left(u_{1}\right)$.

- The inverse is given by projection onto $X_{1}$, hence is continuous.

We remark that the "sufficiently small neighborhood" refers to the fact that we need $\psi$ to exist, and it happens on a sufficiently small neighborhood of zero.

We are now in position to prove each assertion.

(i) Let $h$ be a tangent vector. Then there is a curve $u(t)$ in $M$ with $u(0)=u_{0}$ and $u^{\prime}(0)=h$. Now, since $u(t)$ lies in $M$ we have that

$$
\begin{aligned}
G(u(t))=0 & \Longrightarrow G^{\prime}(u(0))\left[u^{\prime}(0)\right]=G^{\prime}\left(u_{0}\right)[h]=0 \\
& \Longrightarrow h \in \operatorname{ker} G^{\prime}\left(u_{0}\right) .
\end{aligned}
$$

Conversely, if $G^{\prime}\left(u_{0}\right)[h]=0$ then $t h$ is in the domain of $\varphi$ and $u(t)=\varphi(t h)$ is an admissible curve. Indeed, $G(u(t))=0, u(0)=0$ and

$$
u^{\prime}(0)=\varphi^{\prime}(0)[h]=I[h]=h .
$$

We have thus proved that the tangent space at $u_{0}$ is indeed a vector space, precisely the kernel of $G^{\prime}\left(u_{0}\right)$. Note that it is closed.

(ii) The construction is essentialy done. It remains to note that if $G$ is of class $C^{k}$ then $F$ is of class $C^{k}$ and consequently $\psi$ and $\varphi$ are of class $C^{k}$.

(iii) Suppose $G$ is a submersion at every point in $M$. Then, by what we have constructed, the tangent space exists and is closed, and every point has a homeomorphism between a neighborhood of zero in the tangent space and a neighborhood of itself. Now, let $u_{0}^{\prime}$ be another point in $M$ with corresponding map $\varphi^{\prime}$. We must show that the coordinate changes are as regular as $G$. Let $u \in M$. Then it has two representations:

$$
\begin{aligned}
& u=\varphi(h), \quad h \in T_{u_{0}} M, \\
& u=\varphi^{\prime}\left(h^{\prime}\right), \quad h \in T_{u_{0}^{\prime}} M .
\end{aligned}
$$

Now, by the definition of $\varphi$ we have that

$$
h=P\left(u-u_{0}\right)=P\left(\varphi^{\prime}\left(h^{\prime}\right)-u_{0}\right)=\varphi^{-1} \circ \varphi^{\prime}\left(h^{\prime}\right) .
$$

Now, if $G$ is of class $C^{k}$ then $\psi$ is of class $C^{k}$, by the inverse function theorem. Then follows that $\varphi^{\prime}$ is of class $C^{k}$. Since $P$ is linear, it is infinitely differentiable. Hence $\varphi^{-1} \circ \varphi^{\prime}$ is of class $C^{k}$.

This completes the proof. 


\subsection{The Theorem of Lagrange Multipliers}

Let $J: X \longrightarrow \mathbb{R}$ be a $C^{1}$ functional.

Definition 5.2.1. The mapping

$$
\left.J^{\prime}(u)\right|_{T_{u} M}: h \in T_{u} M \mapsto J^{\prime}(u)[h] \in \mathbb{R}
$$

is the restriction of $J^{\prime}(u)$ to the tangent space $T_{u} M$. The point $u \in M$ is said to be a critical point of $\left.J\right|_{M}$ if

$$
\left.J\right|_{M} ^{\prime}=\left.J^{\prime}(u)\right|_{T_{u} M}=0
$$

Theorem 5.2.2. Let $G: \Omega \subset X \longrightarrow Y$ be a submersion at $u_{0} \in M=G^{-1}(0)$ and let $f: X \longrightarrow Z$ be differentiable at $u_{0}$, where $Z$ is another Banach space. Then the following assertions are equivalent

(i) $u_{0}$ is a critical point of $\left.f\right|_{M}$.

(ii) There exists $\Lambda_{u_{0}} \in \mathcal{L}(Y, Z)$ such that $u_{0}$ is a critical point of

$$
f-\Lambda_{u_{0}} \circ G: X \longrightarrow Z
$$

that is,

$$
f^{\prime}\left(u_{0}\right)[v]-\Lambda_{u_{0}}\left(G^{\prime}\left(u_{0}\right)[v]\right)=0 \forall v \in X
$$

Moreover, if one of the above holds true then the function $\Lambda_{u_{0}}$ is unique, and it is called the Lagrange Multiplier.

Proof. First we show that (i) $\Longrightarrow$ (ii). We have

$$
X=\operatorname{ker} G^{\prime}\left(u_{0}\right) \oplus(I-P)(X) .
$$

To simplify the notation, we denote

$$
Q:=I-P, \quad L:=(I-P)(X)=\operatorname{Im} Q .
$$

Note that

$$
H_{u_{0}}:=\left.G^{\prime}\left(u_{0}\right)\right|_{L}: L \longrightarrow Y
$$

is a linear and continuous isomorphism. Define

$$
K_{u_{0}}: v \in L=\operatorname{Im} Q \mapsto f^{\prime}\left(u_{0}\right)[u] \in Z \text { such that } v=Q u
$$

We remark that $K_{u_{0}}$ is well defined: let $v=Q u_{1}=Q u_{2}$. Then

$$
u_{1}-u_{2} \in \operatorname{ker} Q=\operatorname{ker} G^{\prime}\left(u_{0}\right) \subset \operatorname{ker} f^{\prime}\left(u_{0}\right)
$$

and thus $f^{\prime}\left(u_{0}\right)\left[u_{1}\right]=f^{\prime}\left(u_{0}\right)\left[u_{2}\right]$.

If $v \in L$ we have that

$$
v=Q u \text { for some } u \in X, \quad u=P u+Q u=P u+v \text {. }
$$


Therefore,

$$
\begin{aligned}
K_{u_{0}}(v) & =f^{\prime}\left(u_{0}\right)[P u+v] \\
& =f^{\prime}\left(u_{0}\right)[v]
\end{aligned}
$$

and we conclude that

$$
K_{u_{0}}=\left.f^{\prime}\left(u_{0}\right)\right|_{L}
$$

is linear and continuous.

Define

$$
\Lambda_{u_{0}}=K_{u_{0}} \circ H_{u_{0}}: Y \longrightarrow Z \text {. }
$$

It is linear and continuous, as a composition of linear and continuous maps. Moreover, for each $v \in X$ we have that

$$
\begin{aligned}
\Lambda_{u_{0}}\left(G^{\prime}\left(u_{0}\right)[v]\right) & =\Lambda_{u_{0}}\left(G^{\prime}\left(u_{0}\right)[P u+Q u]\right) \\
& =\Lambda_{u_{0}}\left(G^{\prime}\left(u_{0}\right)[Q u]\right) \\
& =\Lambda_{u_{0}}\left(H_{u_{0}}(Q u)\right) \\
& =K_{u_{0}}(Q u) \\
& =f^{\prime}\left(u_{0}\right)[Q u] \\
& =f^{\prime}\left(u_{0}\right)[u] .
\end{aligned}
$$

We now prove that (ii) $\Longrightarrow$ (i). It suffices to note that if $h \in T_{u_{0}} M=\operatorname{ker} G^{\prime}\left(u_{0}\right)$ then

$$
f^{\prime}\left(u_{0}\right)[h]=\Lambda_{u_{0}}\left(G^{\prime}\left(u_{0}\right)[h]\right)=0 .
$$

Finally, we show that if (i) or (ii) holds then $\Lambda_{u_{0}}$ is unique. Suppose that given $u_{0}$ there are $\Lambda_{u_{0}}$ and $\tilde{\Lambda}_{u_{0}}$. Given $y \in Y$, there is $u \in X$ such that

$$
y=G^{\prime}\left(u_{0}\right)[u]
$$

(since $G^{\prime}\left(u_{0}\right)$ is surjective). Then

$$
\begin{aligned}
\Lambda_{u_{0}}(y) & =\Lambda_{u_{0}}\left(G^{\prime}\left(u_{0}\right)[u]\right) \\
& =\Lambda_{u_{0}}\left(G^{\prime}\left(u_{0}\right)[Q u]\right) \\
& =f^{\prime}\left(u_{0}\right)[Q u] \\
& =\tilde{\Lambda}_{u_{0}}(y),
\end{aligned}
$$

which completes the proof.

The next example will be useful afterwards.

Example 5.2.3. Suppose $Y=\mathbb{R}^{2}$ and $Z=\mathbb{R}$. Then

$$
G^{\prime}\left(u_{0}\right)[v]=\left[\begin{array}{l}
G_{1}^{\prime}\left(u_{0}\right)[v] \\
G_{2}^{\prime}\left(u_{0}\right)[v]
\end{array}\right]
$$

and

$$
\Lambda_{u_{0}}=\left[\begin{array}{ll}
\lambda_{1} & \lambda 2
\end{array}\right]
$$


hence

$$
\Lambda_{u_{0}}\left(G^{\prime}\left(u_{0}\right)[v]\right)=\left[\begin{array}{ll}
\lambda_{1} & \lambda 2
\end{array}\right]\left[\begin{array}{l}
G_{1}^{\prime}\left(u_{0}\right)[v] \\
G_{2}^{\prime}\left(u_{0}\right)[v]
\end{array}\right]=\lambda_{1} G_{1}^{\prime}\left(u_{0}\right)[v]+\lambda_{2} G_{2}^{\prime}\left(u_{0}\right)[v],
$$

which completes the example. 


\section{Chapter 6}

\section{Proof of the main result}

We are now in position to prove Theorem 1.0.1.

\subsection{An auxiliary problem}

Our aim is to build a functional whose critical points will be the weak solutions to the problem. We want to deal with homogeneous boundary conditions. This will help us build the functional in a more convenient way.

Let

$$
\alpha=\int_{\partial \Omega} h_{2} d s-\int_{\partial \Omega} h_{1} d s .
$$

Consider the following auxiliary problem:

$$
\begin{aligned}
\Delta^{2} \chi-\Delta \chi & =\alpha /|\Omega| \quad \text { in } \quad \Omega, \\
\frac{\partial \chi}{\partial \mathbf{n}} & =h_{1} \quad \text { on } \quad \partial \Omega, \\
\frac{\partial \Delta \chi}{\partial \mathbf{n}} & =h_{2} \quad \text { on } \quad \partial \Omega . \\
\int_{\Omega} \chi d x & =0 .
\end{aligned}
$$

We make the following change of variables:

$$
\theta=\Delta \chi
$$

By Theorem A.1.1 we know that there is a unique weak solution to

$$
\begin{aligned}
\Delta \theta-\theta & =\alpha /|\Omega| \quad \text { in } \quad \Omega \\
\frac{\partial \theta}{\partial \mathbf{n}} & =h_{2} \quad \text { on } \quad \partial \Omega
\end{aligned}
$$

and the solution satisfies

$$
\int_{\Omega} \theta d x=\int_{\partial \Omega} h_{1} d s
$$


by the way we have defined $\alpha$. Then, by Theorem A.1.2 there is a unique weak solution to

$$
\begin{aligned}
\Delta \chi & =\theta, \quad & \text { in } \Omega \\
\frac{\partial \chi}{\partial \mathbf{n}} & =h_{1} \quad & \text { in } \quad \partial \Omega \\
\int_{\Omega} \chi d x & =0 &
\end{aligned}
$$

and such solution is the solution to the auxiliary problem. Note that the solution is unique. Indeed, let $\chi_{1}, \chi_{2}$ be two solutions to (6.8)-(6.10). Then $w=\chi_{1}-\chi_{2}$ satisfies

$$
\Delta w=0 \text { in } \Omega, \quad \frac{\partial w}{\partial \mathbf{n}}=0 \text { on } \partial \Omega .
$$

Then multiplying by $w$ and integrating we have

$$
\int_{\Omega} w \Delta w d x=0 \Longrightarrow \int_{\Omega}|\nabla w|^{2} d x+\int_{\partial \Omega} w \frac{\partial w}{\partial \mathbf{n}} d s=0
$$

hence $\nabla w=0$ and thus $w$ is a constant. Hence, there is uniqueness up to a constant. Then the hypothesis $\int_{\Omega} \chi d x=0$ implies uniqueness.

Set

$$
\varphi=\phi-\chi-\mu
$$

where

$$
\mu=\frac{1}{|\Omega|} \int_{\Omega} \phi d x
$$

With the new variables $(u, \omega, \varphi, \mu)$ our problem becomes

$$
\begin{aligned}
-\Delta u+q(\chi+\varphi) u & =\omega u-\mu q u \quad \text { in } \Omega, \\
\Delta^{2} \varphi-\Delta \varphi & =q u^{2}-\alpha /|\Omega| \quad \text { in } \Omega, \\
u & =0 \quad \text { on } \partial \Omega, \\
\int_{\Omega} u^{2} d x & =1, \\
\frac{\partial \varphi}{\partial \mathbf{n}} & =0 \quad \text { on } \partial \Omega, \\
\frac{\partial \Delta \varphi}{\partial \mathbf{n}} & =0 \quad \text { on } \partial \Omega, \\
\int_{\Omega}^{\varphi d x} & =0 .
\end{aligned}
$$

Notice that the compatibility condition between (6.12) and (6.15) and (6.16) now reads as

$$
\int_{\Omega} q u^{2} d x=\alpha
$$


Let

$$
\begin{aligned}
& S:=\left\{u \in H_{0}^{1}(\Omega): \int_{\Omega} u^{2} d x=1\right\}, \\
& N:=\left\{u \in H_{0}^{1}(\Omega): \int_{\Omega} q u^{2} d x=\alpha\right\}, \\
& M:=S \cap N .
\end{aligned}
$$

Notice that $\alpha$ depends on both the boundary conditions to the original problem.

If the problem has a solution, then $M \neq \emptyset$. Hence,

$$
q_{\min } \leq \alpha \leq q_{\max }
$$

where

$$
q_{\min }=\inf _{\Omega} q \quad \text { and } \quad q_{\max }=\sup _{\Omega} q
$$

Indeed, suppose $\alpha<q_{\text {min. }}$. Then

$$
\alpha=\int_{\Omega} q u^{2} d x \geq q_{\min }>\alpha
$$

a contradiction. The case $\alpha>q_{\max }$ is analogous.

From $(6.21)$ we deduce that $q^{-1}(\alpha)$ is not empty, with its measure playing a major role. Suppose $\alpha=q_{\text {min }}$ and $\left|q^{-1}(\alpha)\right|=0$. Then

$$
\int_{\Omega} q u^{2} d x=\int_{\Omega} \chi_{q^{-1}(\alpha)} \alpha u^{2} d x+\int_{\Omega}\left(1-\chi_{q^{-1}(\alpha)}\right) q u^{2} d x>\alpha .
$$

If $\alpha=q_{\max }$ and $\left|q^{-1}(\alpha)\right|=0$ we proceed in an analogous manner to conclude that $M$ is empty and so the problem has no solutions. Therefore, we arrive at the following necessary condition for the existence of solutions: either

$$
q_{\min }<\alpha<q_{\max }
$$

or

$$
\left|q^{-1}(\alpha)\right| \neq 0
$$

\subsection{The manifold $M$}

We first note that $M$ is symmetric with respect to the origin: if $u \in M$ then $-u \in M$. This follows trivially from the definition of $M$.

We also note that $M$ is weakly closed in $H_{0}^{1}(\Omega)$. Indeed, let $\left(u_{n}\right)_{n} \subset M$ such that $u_{n} \rightarrow u$ in $H_{0}^{1}(\Omega)$. Since $H_{0}^{1}(\Omega)$ has a compact embedding into $L^{2}(\Omega)$ it follows that $u_{n}$ converges strongly in $L^{2}(\Omega)$. Then by $\left\|u-u_{n}\right\|_{2} \geq\left|\|u\|_{2}-\left\|u_{n}\right\|_{2}\right|$ and since $\left\|u-u_{n}\right\| \rightarrow 0$ we know that

$$
||\left|u\left\|_{2}-\right\| u_{n} \|_{2}\right| \rightarrow 0
$$


and thus $\|u\|_{2}=1$ and $u \in S$. Now, note that

$$
\int_{\Omega} q u^{2} d x-\int_{\Omega} q u_{n}^{2} d x=\int_{\Omega} q\left(u^{2}-u_{n}^{2}\right) d x \rightarrow 0
$$

by the Dominated Convergence Theorem (note that $u^{2}-u_{n}^{2} \rightarrow 0$ a.e. and that $\left|u^{2}-u_{n}^{2}\right| \leq$ $\left.u^{2}+u_{n}^{2} \in L^{1}(\Omega)\right)$.

Now, we want to show that under condition (6.22) the set $M$ is not empty. For this, we introduce the following notation.

Let $A \subset \Omega$ be an open subset,

$$
S_{A}:=\left\{u \in H_{0}^{1}(A): \int_{A} u^{2} d x=1\right\}
$$

and

$$
g_{A}: u \in S_{A} \mapsto \int_{A} q u^{2} d x \in \mathbb{R}
$$

It is immediately seen that.

$$
g_{A}\left(S_{A}\right) \subset\left[\inf _{A} q, \sup _{A} q\right] .
$$

Lemma 6.2.1. The following inclusion holds:

$$
\left(\inf _{A} q, \sup _{A} q\right) \subset \overline{g_{A}\left(S_{A}\right)}
$$

Proof. The idea is to take a number in $\left(\inf _{A} q, \sup _{A} q\right)$ and show that it is the limit of a sequence $\left(g_{A}\left(u_{n}\right)\right)$.

Let $d \in\left(\inf _{A} q, \sup _{A} q\right)$ and $y, z \in A$ such that

$$
q(y)<d<q(z)
$$

Since intervals on the real line are convex, there exists a $\lambda \in(0,1)$ such that

$$
d=(1-\lambda) q(y)+\lambda q(z) .
$$

From the existence of cutoff functions we have that there exist two smooth functions $v, w$ supported on disjoint balls centered in $y$ and $z$, respectively, and contained in $A$, such that

$$
\int_{A} v^{2} d x=1-\lambda, \quad \int_{A} w^{2} d x=\lambda
$$

For $n \in \mathbb{N}$ define

$$
v_{n}(x)=n^{3 / 2} v(n(x-y)+y)
$$

and

$$
w_{n}(x)=n^{3 / 2} w(n(x-z)+z) .
$$

Note that $\operatorname{supp} v_{1}=\operatorname{supp} v$ and analogously for $w, w_{1}$, and that the supports shrink as $n$ increases. Thus for every $n \in \mathbb{N}$ the functions $v_{n}, w_{n}$ have disjoint supports. Also note that

$$
\int_{A} v_{n}^{2} d x=1-\lambda, \quad \int_{A} w_{n}^{2} d x=\lambda
$$


Define

$$
u_{n}:=v_{n}+w_{n} .
$$

By the above, we have that $u_{n} \in S_{A}$ for all $n \in \mathbb{N}$.

Now, we note that

$$
\begin{aligned}
\lim _{n \rightarrow \infty} g_{A}\left(v_{n}\right) & =\lim _{n \rightarrow \infty} \int_{A} q(x) n^{3} v^{2}(n(x-y)+y) d x \\
& =\lim _{n \rightarrow \infty} \int_{A} q\left(\frac{t-y}{n}+y\right) v^{2}(t) d t \\
& =(1-\lambda) q(y)
\end{aligned}
$$

by the Dominated Convergence Theorem (note that all functions are bounded, as well as the domain of integration). Analogously,

$$
\lim _{n \rightarrow \infty} \int_{A} g_{A}\left(w_{n}\right) d x=\lambda q(z)
$$

It follows at once that

$$
\lim _{n \rightarrow \infty} g_{A}\left(u_{n}\right)=d
$$

and so the proof is complete.

We can conclude:

Proposition 6.2.2. Let $A \subset \Omega$ be an open subset. If $\alpha \in\left(\inf _{A} q, \sup _{A} q\right)$ then there exists $u \in H_{0}^{1}(A)$ such that

$$
\int_{A} u^{2} d x=1 \quad \text { and } \quad \int_{A} q u^{2} d x=\alpha .
$$

Proof. From inclusions (6.24) and (6.25) we have that

$$
\left[\inf _{A} q, \sup _{A} q\right]=\overline{g_{A}\left(S_{A}\right)} .
$$

Note that $S_{A}$ is connected. Indeed, consider the following map:

$$
\begin{aligned}
F: H_{0}^{1}(A) \backslash\{0\} & \longrightarrow S_{A} \\
u & \longmapsto \frac{u}{\|u\|_{2}} .
\end{aligned}
$$

Of course $F$ is continuous and takes the connected set $L^{2}(A) \backslash\{0\}$ onto $S_{A}$. Since the image of a connected set via a continuous function is connected, the claim follows.

Moreover, since $g_{A}$ is continuous we have that

$$
\left(\inf _{A} q, \sup _{A} q\right) \subset g_{A}\left(S_{A}\right) .
$$

The claim is now immediate.

Theorem 6.2.3. Assume that $\inf _{\Omega} q<\alpha<\sup _{\Omega} q$. Then $M$ is not empty.

Proof. Take $A=\Omega$ and use the above proposition.

Note that $M$ has subsets of arbitrarily large genus: 
Theorem 6.2.4. Let $u_{1}, \ldots, u_{k} \in M$ be functions with disjoint supports and let

$$
V_{k}=\left\langle u_{1}, \ldots, u_{k}\right\rangle
$$

be the space spanned by $u_{1}, \ldots, u_{k}$. Then $M \cap V_{k}$ is the $(k-1)$-dimensional sphere, hence $\gamma\left(M \cap V_{k}\right)=k$.

Proof. Since the supports are disjoint the functions $u_{1}, \ldots, u_{k}$ are all linearly independent and hence the space $V_{k}$ has dimension $k$. Notice that $S$ is the $L^{2}$-sphere in $H_{0}^{1}(\Omega)$, so $S \cap V_{k}$ is just the $L^{2}$-sphere in $V_{k}$. Hence we need to show that

$$
M \cap V_{k}=S \cap V_{k}
$$

Now, of course

$$
M \cap V_{k} \subset S \cap V_{k} .
$$

On the other hand, let $u=\sum_{1}^{k} \alpha_{i} u_{i} \in S \cap V_{k}$. Then

$$
1=\|u\|_{2}^{2}=\sum_{1}^{k} \alpha_{i}^{2}
$$

Therefore

$$
\int_{\Omega} q u^{2} d x=\int_{\Omega} q \sum_{1}^{k} \alpha_{i}^{2} u_{i}^{2} d x=\sum_{1}^{k} \alpha_{i}^{2} \int_{\Omega} q u_{i}^{2} d x=\alpha \sum_{1}^{k} \alpha_{i}^{2}=\alpha,
$$

hence $u \in M \cap V_{k}$.

Now, it is natural if one raises the question of whether there exists such functions with disjoint supports for arbitrary $k$. The answer is positive:

Theorem 6.2.5. If (6.22) holds then for every $k \geq 2$ there exist $k$ functions $u_{1}, \ldots, u_{k} \in M$ with disjoint supports. Hence $\gamma(M)=+\infty$.

Proof. By hypothesis, the sets

$$
\Omega_{+}:=\{x \in \Omega: q(x)>\alpha\}, \quad \Omega_{-}:=\{x \in \Omega: q(x)<\alpha\}
$$

are open and non-empty. There exists $2 k$ disjoint balls $Y_{1}, \ldots, Y_{k} \subset \Omega_{-}$and $Z_{1}, \ldots, Z_{k} \subset \Omega_{+}$. Let

$$
A_{i}=Y_{i} \cup Z_{i}, \quad i=1, \ldots, k .
$$

Then, by construction,

$$
\inf _{A_{i}} q<\alpha<\sup _{A_{i}} q
$$

Then, by Proposition 6.2.2 for each $i$ there exists $u_{i} \in H_{0}^{1}\left(A_{i}\right)$ such that

$$
\int_{\Omega} u_{i}^{2} d x=\int_{A_{i}} u_{i}^{2} d x=1 \quad \text { and } \quad \int_{\Omega} q u_{i}^{2} d x=\int_{A_{i}} q u_{i}^{2} d x=\alpha .
$$

All the functions $u_{i}$ have disjoint supports and belong to $M$.

That $\gamma(M)=+\infty$ follows immediately by the subaditivity property of the genus. 
Let

$$
\begin{aligned}
& G_{1}: u \in H_{0}^{1}(\Omega) \mapsto \int_{\Omega} u^{2} d x-1 \in \mathbb{R}, \\
& G_{2}: u \in H_{0}^{1}(\Omega) \mapsto \int_{\Omega} q u^{2} d x-\alpha \in \mathbb{R}
\end{aligned}
$$

and

$$
G=\left(G_{1}, G_{2}\right)
$$

Then

$$
M=\left\{u \in H_{0}^{1}(\Omega): G_{1}(u)=G_{2}(u)=0\right\}=G^{-1}(0) .
$$

We note that $G$ is of class $C^{1}$, for

$$
G_{1}^{\prime}(u)[v]=2 \int_{\Omega} u v d x, \quad v \in H_{0}^{1}(\Omega)
$$

Note that $G_{1}^{\prime}$ is linear. Let us show that $G_{1}^{\prime}$ is continuous. Let $\left(u_{n}\right) \subset H_{0}^{1}(\Omega)$ be such that $u_{n} \rightarrow u \in H_{0}^{1}(\Omega)$. Then $\left(u_{n}\right)$ also converges in $L^{2}(\Omega)$. Hence, for all $v \in H_{0}^{1}(\Omega)$ we have

$$
\begin{aligned}
\left\|G_{1}^{\prime}\left(u_{n}\right)-G_{1}^{\prime}(u)\right\| & =\sup _{\|v\|_{H_{0}^{1}(\Omega)}=1}\left|G_{1}^{\prime}\left(u_{n}-u\right)[v]\right| \\
& =\sup _{\|v\|_{H_{0}^{1}(\Omega)}=1}\left|\int_{\Omega}\left(u_{n}-u\right) v d x\right| \\
& \leq \sup _{\|v\|_{H_{0}^{1}(\Omega)}=1}\left\|u_{n}-u\right\|_{2}\|v\|_{2} \\
& \leq c\left\|u_{n}-u\right\|_{2} \rightarrow 0
\end{aligned}
$$

By an analogous argument we see that $G_{2}^{\prime}$ is continuous as well. Thus, $G_{1}$ and $G_{2}$ are of class $C^{1,1}$. Now, $G^{\prime}$ is linear and continuous, because its components are continuous. Hence $G$ is $C^{1,1}$ as well.

If we show that $G_{1}^{\prime}(u)$ and $G_{2}^{\prime}(u)$ are linearly independent, then $G$ will be a submersion and $M$ a submanifold (of codimension 2).

Proposition 6.2.6. Assume $M$ is not empty. The differentials $G_{1}^{\prime}(u)$ and $G_{2}^{\prime}(u)$ are linearly independent for every $u \in M$ if and only if

$$
\left|q^{-1}(\alpha)\right|=0
$$

Proof. First, assume (6.28). We will show that $G_{1}^{\prime}(u)$ and $G_{2}^{\prime}(u)$ are linearly independent, for all $u \in M$. Suppose that there are $a, b \in \mathbb{R}$ such that

$$
a G_{1}^{\prime}(u)+b G_{2}^{\prime}(u)=0 \quad \text { in } H^{-1}(\Omega)
$$

for some $u \in M$. Evaluating this expression in $u$, we find that $a+b \alpha=0$. Then

$$
a G_{1}^{\prime}(u)[v]+b G_{2}^{\prime}(u)[v]=b\left(-\alpha \int_{\Omega} u v d x+\int_{\Omega} q u v d x\right)=0 \quad \forall v \in H_{0}^{1}(\Omega),
$$

that is,

$$
b \int_{\Omega}(q-\alpha) u v d x=0 \quad \forall v \in H_{0}^{1}(\Omega)
$$


If $b \neq 0$ then we would have $(q-\alpha) u=0$ a.e., and hence, in view of (6.28), $u=0$, a contradiction. Thus $G_{1}^{\prime}(u)$ and $G_{2}^{\prime}(u)$ are linearly independent for all $u \in M$.

Now, suppose (6.28) is not satisfied. Then $q^{-1}(\alpha)$ has not empty interior, hence there is some test function $u$ with support in $q^{-1}(\alpha)$ such that $\|u\|_{2}=1$. It is immediately seen that $u \in M$ because $q u=\alpha u$ and hence

$$
G_{2}^{\prime}(u)=\alpha G_{1}^{\prime}(u)
$$

which completes the proof.

\subsection{Existence of solutions}

We now proceed to study the variational framework of the problem. Our aim is to construct a functional whose critical points will be the weak solutions of the problem.

Following Gazzola et al. (2010), let

$$
V=\left\{\xi \in H^{2}(\Omega): \frac{\partial \xi}{\partial \mathbf{n}}=0 \text { on } \partial \Omega\right\} .
$$

We remark that $V$ is a closed subspace of $H^{2}(\Omega)$. Indeed, let $\left(v_{n}\right) \subset V$ such that $v_{n} \rightarrow v$ in $\mathrm{V}$. Then $0=\gamma_{1}\left(v_{n}\right) \rightarrow \gamma_{1}(v)$ and hence $\gamma_{1}(v)=0$, where $\gamma_{1}$ denotes the trace operator which for smooth functions gives the directional derivative on the boundary. Being a closed subspace, $V$ inherits the Hilbert space structure of $H^{2}(\Omega)$.

Recall that

$$
\varphi=\phi-\chi-\mu
$$

where

$$
\mu=\frac{1}{|\Omega|} \int_{\Omega} \phi d x
$$

In this way, we have $\bar{\varphi}=0$. Consider then the following decomposition of $V$ :

$$
V=\tilde{V} \oplus \mathbb{R}
$$

where

$$
\tilde{V}=\{\eta \in V: \bar{\eta}=0\} .
$$

On $\tilde{V}$ we have the equivalent norm

$$
\|\eta\|_{\tilde{V}}=\left(\|\nabla \eta\|_{2}^{2}+\|\Delta \eta\|_{2}^{2}\right)^{1 / 2}
$$

Consider the functional $F: H_{0}^{1}(\Omega) \times H^{2}(\Omega)$ defined below:

$$
\begin{aligned}
F(u, \varphi)= & \frac{1}{2} \int_{\Omega}|\nabla u|^{2} d x+\frac{1}{2} \int_{\Omega} q(\varphi+\chi) u^{2} d x-\frac{1}{4} \int_{\Omega}(\Delta \varphi)^{2} d x \\
& -\frac{1}{4} \int_{\Omega}|\nabla \varphi|^{2} d x-\frac{\alpha}{2|\Omega|} \int_{\Omega} \varphi d x .
\end{aligned}
$$

It is easy to see that this functional is of class $C^{1}$ and that for every $u \in H_{0}^{1}(\Omega)$ and 
$\varphi \in H^{2}(\Omega)$ we have

$$
\begin{aligned}
& F_{u}^{\prime}(u, \varphi)[v]=\int_{\Omega} \nabla u \cdot \nabla v d x+\int_{\Omega} q(\varphi+\chi) u v d x \\
& F_{\varphi}^{\prime}(u, \varphi)[\xi]=\frac{1}{2} \int_{\Omega} q \xi u^{2} d x-\frac{1}{2} \int_{\Omega} \Delta \varphi \Delta \xi d x-\frac{1}{2} \int_{\Omega} \nabla \varphi \cdot \nabla \xi d x-\frac{\alpha}{2|\Omega|} \int_{\Omega} \xi d x
\end{aligned}
$$

for every $v \in H_{0}^{1}(\Omega)$ and $\xi \in H^{2}(\Omega)$.

Theorem 6.3.1. Let $(u, \varphi) \in H_{0}^{1}(\Omega) \times H^{2}(\Omega)$. Then there exist $\omega, \mu \in \mathbb{R}$ such that $(u, \varphi, \omega, \mu)$ is a solution to (6.11)-(6.17) if and only if $(u, \varphi)$ is a critical point of $F$ constrained on $M \times \tilde{V}$, in which case the real constants $\omega, \mu$ are the two Lagrange multipliers with respect to $F_{u}^{\prime}$.

Proof. Note that $(u, \varphi, \omega, \mu)$ is a weak solution to (6.11)-(6.17) if $(u, \varphi) \in M \times \tilde{V}$ and

$$
\begin{aligned}
& \forall v \in H_{0}^{1}(\Omega) F_{u}^{\prime}(u, \varphi)[v]=\omega \int_{\Omega} u v d x-\mu \int_{\Omega} q u v d x \\
& \forall \xi \in V F_{\varphi}^{\prime}(u, \varphi)[\xi]=0 .
\end{aligned}
$$

Now, $(u, \varphi)$ is a critical point of $F$ constrained on $M \times \tilde{V}$ if

$$
\begin{aligned}
& \forall v \in T_{u} M F_{u}^{\prime}(u, \varphi)[v]=0, \\
& \forall \xi \in \tilde{V} F_{\varphi}^{\prime}(u, \varphi)[\xi]=0 .
\end{aligned}
$$

Note that the tangent space to $\tilde{V}$ at $\varphi$ is $\tilde{V}$ itself.

Recall that

$$
T_{u} M=\left\{v \in H_{0}^{1}(\Omega): G^{\prime}(u)[v]=0\right\}
$$

But

$$
G^{\prime}(u)[v]=\left(2 \int_{\Omega} u v d x, 2 \int_{\Omega} q u v d x\right)=0 \Longleftrightarrow \int_{\Omega} u v d x=\int_{\Omega} q u v d x=0
$$

Now, it is easy to see that a weak solution is a constrained critical point. Suppose that $(u, \varphi)$ is a constrained critical point. Then, by the theorem of Lagrange multipliers we have that

$$
F_{u}^{\prime}(u, \varphi)[v]=\omega \int_{\Omega} u v d x-\mu \int_{\Omega} q u v d x
$$

It remains to prove that $F_{\varphi}^{\prime}(u, \varphi)[\xi]=0 \forall \xi \in V$. But this follows from decomposition (6.29). Note that $F_{\varphi}^{\prime}(u, \varphi)[r]=0$ for every constant $r \in \mathbb{R}$.

The functional $F$ constrained on $M \times \tilde{V}$ is unbounded from above and from below. Indeed, for $\varphi=0$ we have

$$
F(u, 0)=\frac{1}{2} \int_{\Omega}|\nabla u|^{2} d x+\frac{1}{2} \int_{\Omega} q \chi u^{2} d x
$$

which is unbounded from above as $\|\nabla u\|_{2} \rightarrow+\infty$. On the other hand, let $u, \varphi$ be fixed. Note that for all $t>0$ we have $t \varphi \in \tilde{V}$. Then

$$
\begin{aligned}
F(u, t \varphi) & =\|\nabla u\|_{2}+t \frac{1}{2} \int_{\Omega} q \varphi u^{2} d x+\int_{\Omega} q \chi u^{2} d x-t^{2} \frac{1}{4} \int_{\Omega}(\Delta \varphi)^{2} d x-t^{2} \frac{1}{4} \int_{\Omega}|\nabla \varphi|^{2} d x \\
& =c_{1}+t c_{2}-t^{2} c_{3} \stackrel{t \rightarrow+\infty}{\longrightarrow}-\infty .
\end{aligned}
$$


Note that $c_{3}>0$.

The unboundedness is a serious difficulty. This issue has been addressed by Benci and Fortunato (1998) and in many subsequent papers. Their standard method goes as follows:

(i) For every fixed $u$ there exists a unique $\Phi(u)$ such that $F_{\varphi}^{\prime}(u, \Phi(u))=0$.

(ii) The map $u \mapsto \Phi(u)$ is of class $C^{1}$.

(iii) The graph of $\Phi$ is a manifold, and we are reduced to study the functional $J(u)=$ $F(u, \Phi(u)$, possibly constrained.

The method sketched above fails in our situation, for two reasons. First, we see that $F_{\varphi}^{\prime}(u, \varphi)=0$ with $\varphi \in \tilde{V}$ is just

$$
\begin{aligned}
\Delta^{2} \varphi-\Delta \varphi-q u^{2}+\alpha /|\Omega| & =0 & & \text { in } \Omega, \\
\frac{\partial \varphi}{\partial \mathbf{n}} & =0 & & \text { on } \partial \Omega, \\
\frac{\partial \Delta \varphi}{\partial \mathbf{n}} & =0 & & \text { on } \quad \partial \Omega, \\
\int_{\Omega} \varphi d x & =0 . & &
\end{aligned}
$$

The problem above has a unique solution if and only if $u \in N$ (one can see this by integrating the equations and using Green's formula). Also, since $N$ is not a manifold (unless $\alpha \neq 0$ ) we cannot require the map $\Phi: u \mapsto \Phi(u)$ to be of class $C^{1}$. We shall then extend such a map $\Phi$.

Proposition 6.3.2. For every $w \in L^{6 / 5}(\Omega)$ there exists a unique $L(w) \in \tilde{V}$ solution of

$$
\begin{aligned}
\Delta^{2} \varphi-\Delta \varphi-w+\bar{w} & =0 & & \text { in } \quad \Omega, \\
\frac{\partial \varphi}{\partial \boldsymbol{n}} & =0 & & \text { on } \quad \partial \Omega, \\
\frac{\partial \Delta \varphi}{\partial \boldsymbol{n}} & =0 & & \text { on } \quad \partial \Omega, \\
\int_{\Omega} \varphi d x & =0 . & &
\end{aligned}
$$

The map $L: L^{6 / 5}(\Omega) \longrightarrow \tilde{V}$ is linear and continuous, hence of class $C^{\infty}$.

Proof. We begin by noting that $\tilde{V}$ is a Hilbert space. Indeed, let $\left(u_{n}\right) \subset \tilde{V}$ be a Cauchy sequence. Then it is a Cauchy sequence in $H^{2}(\Omega)$, which is a Hilbert space, and thus $u_{n} \rightarrow$ $u \in H^{2}(\Omega)$. But

$$
0=\int_{\Omega} u_{n} d x \rightarrow \int_{\Omega} u d x
$$

and hence $u \in \tilde{V}$.

Now, weak solutions to the problem are functions $\varphi \in \tilde{V}$ such that

$$
\int_{\Omega} \Delta \varphi \Delta v d x+\int_{\Omega} \nabla \varphi \cdot \nabla v d x-\int_{\Omega} w v d x=0 \quad \forall v \in \tilde{V}
$$

Consider then the bilinear form $b: \tilde{V} \times \tilde{V} \longrightarrow \mathbb{R}$ given by

$$
b(\varphi, v)=\int_{\Omega} \Delta \varphi \Delta v d x+\int_{\Omega} \nabla \varphi \cdot \nabla v d x .
$$


This bilinear form actually gives the scalar product in $\tilde{V}$ so it suffices to apply the Riesz Theorem.

Proposition 6.3.3. The map

$$
u \in L^{6}(\Omega) \mapsto q u^{2} \in L^{6 / 5}(\Omega)
$$

is of class $C^{1}$.

Proof. See Example 2.1.18.

As a consequence of the previous propositions, we can define the following map:

$$
\Phi: u \in H_{0}^{1}(\Omega) \mapsto L\left(q u^{2}\right) \in \tilde{V} .
$$

It is clear that

$$
\Phi(u)=\Phi(-u)=\Phi(|u|)
$$

Moreover, for every $(u, \varphi) \in H_{0}^{1}(\Omega) \times \tilde{V}$ we have that $\varphi=\Phi(u)$ if and only if for every $\eta \in \tilde{V}$

$$
\int_{\Omega} \Delta \varphi \Delta \eta d x+\int_{\Omega} \nabla \varphi \nabla \eta d x=\int_{\Omega} q u^{2} \eta d x
$$

Taking $\eta=\Phi(u)$ we see that

$$
\int_{\Omega}(\Delta \Phi(u))^{2} d x+\int_{\Omega}|\nabla \Phi(u)|^{2} d x=\int_{\Omega} q u^{2} \Phi(u) d x
$$

from which we have

$$
\begin{aligned}
\|\Phi(u)\|_{\tilde{V}}^{2} & \leq\|q\|_{\infty} \int_{\Omega} u^{2} \Phi(u) d x \\
& \leq c\left\|u^{2}\right\|_{2}\|\Phi(u)\|_{2} \\
& =c\left(\int_{\Omega} u^{4} d x\right)^{1 / 2}\|\Phi(u)\|_{2} \\
& =c\|u\|_{4}^{2}\|\Phi(u)\|_{2} \\
& \leq c\|u\|_{2}^{2}\|\nabla \Phi(u)\|_{2} \\
& \leq c\|\nabla u\|_{2}^{2}\|\Phi(u)\|_{\tilde{V}}
\end{aligned}
$$

and hence

$$
\|\Phi(u)\|_{\tilde{V}} \leq c\|\nabla u\|_{2}^{2},
$$

that is, $\Phi$ is bounded on bounded sets. We actually have more:

Lemma 6.3.4. The map $\Phi$ is compact. Moreover, if $u_{n} \rightarrow u$ in $H_{0}^{1}(\Omega)$ then

$$
\int_{\Omega} q u_{n}^{2} \Phi\left(u_{n}\right) d x \rightarrow \int_{\Omega} q u^{2} \Phi(u) d x
$$

Proof. Let $u_{n} \rightarrow u$ in $H_{0}^{1}(\Omega)$ and define $B_{n}, B: \tilde{V} \longrightarrow \mathbb{R}$ by

$$
B_{n}(\eta):=\int_{\Omega} q u_{n}^{2} \eta d x, \quad B(\eta):=\int_{\Omega} q u^{2} \eta d x
$$


Such operators are continuous due to Hölder's inequality. For example:

$$
\left|\int_{\Omega} q u^{2} \eta d x\right| \leq\left\|q u^{2}\right\|_{6 / 5}\|\eta\|_{6} \leq\|q\|_{\infty}\|u\|_{4}^{2}\|\eta\|_{2} \leq c|| \nabla \eta\left\|_{2} \leq c\right\| \eta \|_{\tilde{V}}
$$

Recall that $H_{0}^{1}(\Omega) \hookrightarrow L^{r}(\Omega)$ for $r \in[1,6]$, with compact embedding if $r<6$. Hence $u_{n} \rightarrow u$ in $L^{r}(\Omega)$ for all $r \in[1,6]$, hence $\left(u_{n}\right)$, and consequently $\left(u_{n}+u\right)$ are bounded. So $u_{n}^{2} \rightarrow u^{2}$ in $L^{6 / 5}(\Omega)$. Indeed:

$$
\begin{aligned}
\int_{\Omega}\left(u_{n}^{2}-u^{2}\right)^{6 / 5} d x & =\int_{\Omega}\left(u_{n}-u\right)^{6 / 5}\left(u_{n}+u\right)^{6 / 5} d x \\
& \leq\left\|\left(u_{n}-u\right)^{6 / 5}\right\|_{2}\left\|\left(u_{n}+u\right)^{6 / 5}\right\|_{2} \\
& \leq c \int_{\Omega}\left(u_{n}-u\right)^{12 / 5} d x \rightarrow 0
\end{aligned}
$$

From the above it follows that

$$
\begin{aligned}
\left|B_{n}(\eta)-B(\eta)\right| & \leq\|q\|_{\infty}\left\|u_{n}^{2}-u^{2}\right\|_{6 / 5}\|\eta\|_{6} \\
& \leq c\|q\|_{\infty}\left\|u_{n}^{2}-u^{2}\right\|_{6 / 5}\|\eta\|_{\tilde{V}}
\end{aligned}
$$

Hence

$$
\left\|B_{n}-B\right\| \leq \sup _{\eta \neq 0} \frac{c\left\|u_{n}^{2}-u^{2}\right\|_{6 / 5}\|\eta\|_{\tilde{V}}}{\|\eta\|_{\tilde{V}}} \rightarrow 0
$$

and thus $B_{n} \rightarrow B$ in $\mathcal{L}(\tilde{V})$.

On the other hand, we have that $\Phi\left(u_{n}\right) \rightarrow \Phi(u)$ in $\tilde{V}$. Indeed, let $g \in \tilde{V}^{*}$. Then there is some $v_{g} \in \tilde{V}$ such that

$$
g\left(\Phi\left(u_{n}\right)\right)=\int_{\Omega} \nabla \Phi\left(u_{n}\right) \cdot \nabla v_{g} d x+\int_{\Omega} \Delta \Phi(u) \Delta v_{g} d x=\int_{\Omega} q u_{n}^{2} v_{g} d x .
$$

But then

$$
\begin{aligned}
g\left(\phi\left(u_{n}\right)\right)-g(\Phi(u)) & =\int_{\Omega} q\left(u_{n}^{2}-u^{2}\right) v_{g} d x \\
& \leq\|q\|_{\infty}\left\|u_{n}^{2}-u^{2}\right\|_{2}\left\|v_{g}\right\|_{2} \rightarrow 0
\end{aligned}
$$

since $u_{n}^{2} \rightarrow u^{2}$ in $L^{2}(\Omega)$ as well.

We then conclude that

$$
\int_{\Omega} q u_{n}^{2} \Phi\left(u_{n}\right) d x \rightarrow \int_{\Omega} q u^{2} \Phi(u) d x
$$

and hence $\Phi\left(u_{n}\right) \rightarrow \Phi(u)$ in $\tilde{V}$ by $(6.31)$.

Note that for every $u \in N$ we have that $F_{\varphi}^{\prime}(u, \Phi(u))=0$. Indeed, $\Phi(u)$ is the unique solution to the problem in Proposition 6.3.2 with $w=q u^{2}$.

We now define the reduced functional of a single variable:

$$
\begin{aligned}
J: H_{0}^{1}(\Omega) & \longrightarrow \mathbb{R} \\
u & \longmapsto F(u, \Phi(u))
\end{aligned}
$$


With the notation $\varphi_{u}:=\Phi(u)$ the functionl $J$ is explicitly given by

$$
\begin{aligned}
J(u)= & \frac{1}{2} \int_{\Omega}|\nabla u|^{2} d x+\frac{1}{2} \int_{\Omega} q \varphi_{u} u^{2} d x+\frac{1}{2} \int_{\Omega} q \chi u^{2} d x-\frac{1}{4} \int_{\Omega}\left(\Delta \varphi_{u}\right)^{2} d x \\
& -\frac{1}{4} \int_{\Omega}\left|\nabla \varphi_{u}\right|^{2} d x-\frac{\alpha}{2|\Omega|} \int_{\Omega} \varphi_{u} d x \\
= & \frac{1}{2} \int_{\Omega}|\nabla u|^{2} d x+\frac{1}{4} \int_{\Omega}\left(\Delta \varphi_{u}\right)^{2} d x+\frac{1}{4} \int_{\Omega}\left|\nabla \varphi_{u}\right|^{2} d x+\int_{\Omega} q \chi u^{2} d x .
\end{aligned}
$$

We note that $J$ is of class $C^{1}$ on $H_{0}^{1}(\Omega)$ and even. Moreover, for every $u \in M$ we have that

$$
J^{\prime}(u)[v]=F_{u}^{\prime}\left(u, \varphi_{u}\right)[v]+F_{\varphi}^{\prime}\left(u, \varphi_{u}\right)\left[\Phi^{\prime}(u)[v]\right]=F_{u}^{\prime}\left(u, \varphi_{u}\right)[v] \quad \forall v \in H_{0}^{1}(\Omega)
$$

and hence we have the following

Theorem 6.3.5. The pair $(u, \varphi) \in M \times \tilde{V}$ is a critical point of $F$ constrained on $M \times \tilde{V}$ if and only if $u$ is a critical point of $\left.J\right|_{M}$ and $\varphi=\Phi(u)$.

The following lemma gives the existence of solutions to our modified problem.

Lemma 6.3.6. The functional $J$ on $M$ is weakly lower semicontinuous and coercive. In particular, it has a minimum $u \in M$.

Proof. Recall that

$$
J(u)=\frac{1}{2} \int_{\Omega}|\nabla u|^{2} d x+\frac{1}{4} \int_{\Omega}\left(\Delta \varphi_{u}\right)^{2} d x+\frac{1}{4} \int_{\Omega}\left|\nabla \varphi_{u}\right|^{2}+\int_{\Omega} q \chi u^{2} d x
$$

The two terms depending on $\varphi_{u}$ are positive. Also,

$$
\int_{\Omega} q \chi u^{2} d x \geq-\sup _{\Omega}|q| \sup _{\Omega}|\chi|
$$

Hence,

$$
J(u) \geq \int_{\Omega}|\nabla u|^{2} d x-c
$$

and thus $J$ is coercive and bounded from below on $M$.

Now, let $\left(u_{n}\right) \subset M$ such that $u \rightarrow u$. Since $M$ is weakly closed, $u \in M$. By Lemma 6.3 .4 we know that

$$
\frac{1}{4} \int_{\Omega}\left(\Delta \varphi_{u_{n}}\right)^{2} d x+\frac{1}{4} \int_{\Omega}\left|\nabla \varphi_{u_{n}}\right|^{2} d x \rightarrow \frac{1}{4} \int_{\Omega}\left(\Delta \varphi_{u}\right)^{2} d x+\frac{1}{4} \int_{\Omega}\left|\nabla \varphi_{u}\right|^{2} d x .
$$

We also know that $u_{n}^{2} \rightarrow u^{2}$ in $L^{6 / 5}(\Omega)$ so

$$
\begin{aligned}
\int_{\Omega} q \chi\left(u_{n}^{2}-u^{2}\right) d x & \leq c \int_{\Omega} u_{n}^{2}-u^{2} d x \\
& \leq c\left\|u_{n}-u\right\|_{6 / 5} \rightarrow 0 .
\end{aligned}
$$

Finally, the first term is the norm of $u$ in $H_{0}^{1}(\Omega)$, so it is convex and continuous and hence lower semicontinuous. Adding all terms, we have that

$$
J(u) \leq \liminf J\left(u_{n}\right)
$$


and thus $J$ is weakly lower semicontinuous. The existence of the minimum is just given by the Weierstrass theorem.

Remark 6.3.7. Note that $J(u)=J(|u|)$ so the minimum may be assumed to be positive. This completes the first part of Theorem 1.0.1.

\subsection{Multiplicity of solutions}

We will use a deformation argument to show that there are infinitely many solutions. A crucial point is that the functional satisfies the Palais-Smale condition.

Proposition 6.4.1. The functional J satisfies the Palais-Smale condition on $M$.

Proof. Let $\left(u_{n}\right) \subset M$ be a Palais-Smale sequence for $J$. We want to prove that there is some $u_{0} \in M$ such that $u_{n} \rightarrow u_{0}$ in $H_{0}^{1}(\Omega)$. We know that

$$
\left\{J\left(u_{n}\right)\right\} \text { is bounded }
$$

and that

$$
\left.J\right|_{M} ^{\prime}\left(u_{n}\right) \rightarrow 0 .
$$

But (6.33) means precisely that there exists two sequences of real numbers $\left(\alpha_{n}\right),\left(\beta_{n}\right)$ and a sequence $\left(v_{n}\right) \subset H^{-1}$ such that $v_{n} \rightarrow 0$ and

$$
-\Delta u_{n}+q\left(\varphi_{n}+\chi\right) u_{n}=\alpha_{n} u_{n}+\beta_{n} q u_{n}+v_{n}
$$

where $\varphi_{n}:=\varphi_{u_{n}}$.

Since $J$ is coercive and $\left\{J\left(u_{n}\right)\right\}$ is bounded, we know that $\left(u_{n}\right)$ is bounded in $H_{0}^{1}(\Omega)$. Hence there exists $u \in H_{0}^{1}(\Omega)$ such that $u_{n} \rightarrow u$, up to a subsequence. By Lemma 6.3.4 we know that $\varphi_{n} \rightarrow \varphi_{0}$. Also, since $M$ is weakly closed, we know that $u_{0} \in M$. It only remains to show that $u_{n} \rightarrow u_{0}$ in $H_{0}^{1}(\Omega)$.

By (6.34) we have that

$$
\int_{\Omega}\left|\nabla u_{n}\right|^{2} d x+\int_{\Omega} q\left(\varphi_{n}+\chi\right) u_{n}^{2} d x-\left\langle v_{n}, u_{n}\right\rangle=\alpha_{n}+\alpha \beta_{n}
$$

We note that

$$
\left\{\left\|\nabla u_{n}\right\|_{2}\right\} \text { is bounded, } \int_{\Omega} q\left(\varphi_{n}+\chi\right) u_{n}^{2} d x \rightarrow \int_{\Omega} q\left(\varphi_{0}+\chi\right) u_{0}^{2} d x, \quad\left\langle v_{n}, u_{n}\right\rangle \rightarrow 0 .
$$

Indeed,

- The boundedness of $\left(u_{n}\right)$ means precisely that $\left\{\left\|\nabla u_{n}\right\|_{2}\right\}$ is bounded;

- Splitting the integral, we have that the first term converges, by Lemma 6.3.4:

$$
\int_{\Omega} q \varphi_{n} u_{n}^{2} d x \rightarrow \int_{\Omega} q \varphi_{0} u_{0}^{2} d x
$$

Also,

$$
\int_{\Omega} q \chi u_{n}^{2} d x \rightarrow \int_{\Omega} q \chi u_{0}^{2} d x
$$


because, as in the proof of Lemma 6.3.4, $H_{0}^{1}(\Omega) \hookrightarrow L^{6 / 5}$ with compact embedding and so $u_{n}^{2} \rightarrow u_{0}^{2}$ in $L^{6 / 5}(\Omega)$ and hence

$$
\int_{\Omega} q \chi\left(u_{n}^{2}-u_{0}^{2}\right) d x \leq\|q \chi\|_{6}\left\|u_{n}^{2}-u_{0}^{2}\right\|_{6 / 5} \rightarrow 0
$$

- At last,

$$
\left|\left\langle v_{n}, u_{n}\right\rangle\right| \leq\left\|v_{n}\right\|_{H^{-1}}\left\|u_{n}\right\|_{H_{0}^{1}(\Omega)} \leq c\left\|v_{n}\right\|_{H^{-1}} \rightarrow 0
$$

We can then deduce that the right-hand side of (6.35) is bounded. Since bounded sequences of real numbers converge up to subsequences, we can assume that

$$
\alpha_{n}+\alpha \beta_{n}=\lambda+o(1)
$$

with $\lambda \in \mathbb{R}$ and $o(1)$ means that this term goes to zero as $n$ goes to infinity. Then (6.34) becomes

$$
-\Delta u_{n}+q\left(\varphi_{n}+\chi\right) u_{n}-v_{n}=(\lambda+o(1)) u_{n}-\beta_{n}(q-\alpha) u_{n} .
$$

Now, since $u_{0} \in M$ we know that $\left\|u_{0}\right\|_{2}=1$. This, together with the assumption $\left|q^{-1}(\alpha)\right|=0$ implies that $(q-\alpha) u_{0}$ is not identically zero. Then there exists a test function $w_{0} \in C_{c}^{\infty}(\Omega)$ such that

$$
\int_{\Omega}(q-\alpha) u_{0} w_{0} d x \neq 0
$$

Evaluating (6.36) on $w_{0}$ we get

$$
\begin{aligned}
\int_{\Omega} \nabla u_{n} \cdot \nabla w_{0} d x+\int_{\Omega} q\left(\varphi_{n}+\chi\right) u_{n} w_{0} d x-\left\langle v_{n}, w_{0}\right\rangle & -(\lambda+o(1)) \int_{\Omega} u_{n} w_{0} d x \\
& =\beta_{n} \int_{\Omega}(q-\alpha) u_{n} w_{0} d x
\end{aligned}
$$

Note that every term in the left-hand side converges. In particular, we note that

$$
\begin{aligned}
\int_{\Omega} q \varphi_{n} u_{n} w_{0} d x-\int_{\Omega} q \varphi_{0} u_{0} w_{0} d x & \leq c \int_{\Omega} \varphi_{n} u_{n}-\varphi_{0} u_{0} d x \\
& =c \int_{\Omega} \varphi_{n} u_{n}-\varphi_{0} u_{n} d x+c \int_{\Omega} \varphi_{0} u_{n}-\varphi_{0} u_{0} d x \\
& \leq c\left\|\varphi_{n}-\varphi_{0}\right\|+c \int_{\Omega} \varphi_{0} u_{n}-\varphi_{0} u_{0} d x \\
& \rightarrow 0
\end{aligned}
$$

The other terms are continuous linear functionals on $u_{n}$ and thus converge.

Also, by the weak convergence of $\left(u_{n}\right)$,

$$
\int_{\Omega}(q-\alpha) u_{n} w_{0} d x \rightarrow \int_{\Omega}(q-\alpha) u_{0} w_{0} d x
$$

This implies that $\left(\beta_{n}\right)$ is bounded, which in turn implies that $\left(\alpha_{n}\right)$ is bounded. 
Applying (6.36) to $u_{n}-u_{0}$ we get

$$
\begin{array}{r}
\int_{\Omega} \nabla u_{n} \nabla\left(u_{n}-u_{0}\right) d x+\int_{\Omega} q\left(\varphi_{n}+\chi\right) u_{n}\left(u_{n}-u_{0}\right) d x-\left\langle v_{n}, u_{n}-u_{0}\right\rangle \\
=(\lambda+o(1)) \int_{\Omega} u_{n}\left(u_{n}-u_{0}\right) d x+\beta_{n} \int_{\Omega}(q-\alpha) u_{n}\left(u_{n}-u_{0}\right) d x .
\end{array}
$$

Since

$$
\begin{array}{r}
\int_{\Omega} q\left(\varphi_{n}+\chi\right) u_{n}\left(u_{n}-u_{0}\right) d x \rightarrow 0, \quad\left\langle v_{n}, u_{n}-u_{0}\right\rangle \rightarrow 0, \\
(\lambda+o(1)) \int_{\Omega} u_{n}\left(u_{n}-u_{0}\right) d x \rightarrow 0, \quad \beta_{n} \int_{\Omega}(q-\alpha) u_{n}\left(u_{n}-u_{0}\right) d x \rightarrow 0
\end{array}
$$

we conclude that $\left\|\nabla u_{n}\right\|_{2} \rightarrow\left\|\nabla u_{0}\right\|_{2}$ and so $u_{n} \rightarrow u_{0}$ in $H_{0}^{1}(\Omega)$.

Let us show the above convergences. We begin by splitting the first integral. Then, it is immediately seen that

$$
\begin{aligned}
\int_{\Omega} q \chi u_{n}\left(u_{n}-u_{0}\right) d x & \leq c \int_{\Omega} u_{n}\left(u_{n}-u_{0}\right) d x \\
& \leq c\left\|u_{n}\right\|_{2}\left\|u_{n}-u_{0}\right\|_{2} \rightarrow 0
\end{aligned}
$$

since $u_{n} \rightarrow u$ in $L^{2}(\Omega)$. We need to show that

$$
\int_{\Omega} q \varphi_{n} u_{n}\left(u_{n}-u_{0}\right) d x \rightarrow 0
$$

Note that

$$
\begin{aligned}
\int_{\Omega}\left|q \varphi_{n} u_{n}\left(u_{n}-u_{0}\right)\right| d x & \leq\|q\|_{\infty} \int_{\Omega}\left|\varphi_{n} u_{n}\left(u_{n}-u_{0}\right)\right| d x \\
& \leq c\left\|\varphi_{n}\right\|_{2}\left\|u_{n}\left(u_{n}-u_{0}\right)\right\|_{2} \\
& =c\left\|\varphi_{n}\right\|_{2}\left\|u_{n}\right\|\left\|_{4}\right\| u_{n}-u_{0} \|_{4} \\
& \leq c\left\|\varphi_{\tilde{V}}\right\| u_{n}\|\|_{4}\left\|u_{n}-u_{0}\right\|_{4} \rightarrow 0 .
\end{aligned}
$$

As to the second convergence, note that

$$
\left|\left\langle v_{n}, u_{n}-u_{0}\right\rangle\right| \leq\left\|v_{n}\right\| \mid\left\|u_{n}-u_{0}\right\|_{H_{0}^{1}(\Omega)} \rightarrow 0
$$

since $\left(u_{n}-u_{0}\right)$ is bounded in $H_{0}^{1}(\Omega)$.

Regarding the third term, note that

$$
\left|(\lambda+o(1)) \int_{\Omega} u_{n}\left(u_{n}-u_{0}\right) d x\right| \leq c|| u_{n}\left\|_{2}\right\| u_{n}-u_{0} \|_{2} \rightarrow 0
$$

by the compact Sobolev embedding.

The last convergence follows by an argument similar to the one above.

By Theorem 6.2.4 $M$ has compact, symmetric subsets of genus $k$ for every $k \in \mathbb{N}$. Hence, by Theorem 4.6.1 $J$ has infinitely many critical points. The critical points give rise to Lagrange multipliers $\omega_{n}, \mu_{n}$ and then, by the decomposition $\varphi=\phi-\chi-\mu$ to solutions $\left(u_{n}, \omega_{n}, \phi_{n}\right) \in H_{0}^{1}(\Omega) \times \mathbb{R} \times H^{2}(\Omega)$ of the original problem. 
Finally, we show that $\left\|\nabla u_{n}\right\|_{2} \rightarrow+\infty$. We begin by noting that, by Theorem 4.5.5 the critical levels are divergent, that is,

$$
b_{n}=J\left(u_{n}\right)=\frac{1}{2} \int_{\Omega}\left|\nabla u_{n}\right|^{2} d x+\frac{1}{4} \int_{\Omega}\left(\Delta \varphi_{n}\right)^{2} d x+\frac{1}{4} \int_{\Omega}\left|\nabla \varphi_{n}\right|^{2} d x-\frac{1}{2} \int_{\Omega} q \chi u_{n}^{2} d x \rightarrow+\infty .
$$

We proceed, nonetheless, with a direct proof. Since the functional satisfies the PalaisSmale condition and is bounded from below the sublevels have finite genus:

Lemma 6.4.2. For any $b \in \mathbb{R}$ the sublevel

$$
J^{b}=\{u \in M: J(u) \leq b\}
$$

has finite genus.

Proof. We argue by contradiction. Suppose that

$$
D=\left\{b \in \mathbb{R}: \gamma\left(J^{b}\right)=\infty\right\} \neq \emptyset .
$$

Since $\left.J\right|_{M}$ is bounded from below, then $D$ is bounded from below. Then

$$
-\infty<\bar{b}=\inf D<\infty .
$$

Moreover, since $\left.J\right|_{M}$ satisfies the Palais-Smale condition, the set

$$
Z=\left\{u \in M: J(u)=\bar{b},\left.J\right|_{M} ^{\prime}(u)=0\right\}
$$

is compact. Hence there exists a closed symmetric neighborhood $U_{Z}$ of $Z$ such that $\gamma\left(U_{Z}\right)<$ $\infty$. By the Deformation Lemma, there exists an $\varepsilon>0$ such that $J^{\bar{b}-\varepsilon}$ includes a deformation retract of $J^{\bar{b}+\varepsilon} \backslash U_{Z}$. Then, by the properties of the genus,

$$
\gamma\left(J^{\bar{b}+\varepsilon}\right) \leq \gamma\left(J^{\bar{b}+\varepsilon} \backslash U_{Z}\right)+\gamma\left(U_{Z}\right) \leq \gamma\left(J^{\bar{b}-\varepsilon}\right)+\gamma\left(U_{Z}\right)<\infty,
$$

a contradiction.

Let $k \in \mathbb{N}$. By Lemma 6.4.2 there exists some $n \in \mathbb{N}$ depending on $k$ such that

$$
\gamma\left(J^{k}\right)=n
$$

Let

$$
A_{n+1}=\{A \subset M: A=-A, \bar{A}=A, \gamma(A)=n+1\}
$$

By Theorem 6.2.4 we know that $A_{n+1}$ is not empty. By the monotonicity property of the genus, any $A \in A_{n+1}$ is not contained in $J^{k}$, then $\sup _{A} J>k$ and therefore

$$
c_{k}=\inf _{A \in A_{n+1}} \sup _{u \in A} J(u) \geq k
$$

Then $c_{k}$ is a critical value of $\left.J\right|_{M}$. In conclusion: for any $k \in \mathbb{N}$ there is some critical point $u_{k} \in M$ such that $J\left(u_{k}\right) \geq k$. Thus follows the claim about the divergence of critical levels.

Now we note that

$$
\int_{\Omega} q \chi u_{n}^{2} d x \leq\|q \chi\|_{\infty}
$$


so the last term is bounded. Also, from (6.32) we have that

$$
\left\|\varphi_{n}\right\|_{\tilde{V}}=\int_{\Omega}\left(\Delta \varphi_{n}\right)^{2} d x+\int_{\Omega}\left|\nabla \varphi_{n}\right|^{2} d x \leq c|| \nabla u \|_{2}^{2}
$$

and hence $\left\|\nabla u_{n}\right\|_{2} \rightarrow+\infty$.

Remark 6.4.3. We would still get the same results if we perturbed the first equation with a nonlinearity of the form $\kappa|u|^{p-2} u$, for some values of $p$. The appropriate range for $p$ will depend on the sign of the constant $\kappa$. See Pisani and Siciliano (2007) for details. 


\section{Appendix A}

\section{Useful theorems}

In this appendix we collect some theorems that are useful in solving the problem but which we felt did not belong to the main body of the text. They are stated here for the convenience of the reader, and are presented without proof but with due references.

\section{A.1 Neumann boundary value problems}

For the proofs of the following two theorems, see Taylor (1996)

Theorem A.1.1. For $k=0,1, \ldots$, given $f \in H^{k}(\Omega)$ and $g \in H^{k+1 / 2}(\partial \Omega)$ there is a unique solution $u \in H^{k+2}(\Omega)$ to the problem

$$
\begin{aligned}
-\Delta u+u & =f \text { in } \Omega \\
\frac{\partial u}{\partial \boldsymbol{n}} & =g \text { on } \partial \Omega .
\end{aligned}
$$

Theorem A.1.2. For $k=0,1, \ldots, f \in H^{k}(\Omega)$ and $g \in H^{k+1 / 2}(\partial \Omega)$ the problem

$$
\begin{aligned}
& \Delta u=f \text { in } \Omega \\
& \frac{\partial u}{\partial \boldsymbol{n}}=g \text { on } \partial \Omega
\end{aligned}
$$

has a solution $u \in H^{k+2}(\Omega)$ if and only if

$$
\int_{\Omega} f d x=\int_{\partial \Omega} g d s .
$$

\section{A.2 Sobolev spaces}

We present now two important theorems concerning embeddings of Sobolev spaces.

Theorem A.2.1. Let $\Omega \subset \mathbb{R}^{N}$ be a bounded domain with Lipschitz boundary and let $k \geq$ $1,1 \leq p \leq \infty$.

(i) If $k p<N$ then $W^{k, p}(\Omega) \hookrightarrow L^{q}(\Omega)$ for all $1 \leq q \leq N p /(N-k p)$. The embedding is compact provided that $q<N p /(N-k p)$.

(ii) If $k p=n$ then $W^{k, p}(\Omega) \hookrightarrow L^{q}(\Omega)$ for all $1 \leq q<\infty$ and the embedding is compact. 
(iii) If $k p>n$ then $W^{k, p}(\Omega) \hookrightarrow C^{0, \alpha}(\Omega)$ where

$$
\alpha=\left\{\begin{array}{l}
k-n / p \quad \text { if } k-n / p<1 \\
1 \quad \text { if } k-n / p>1
\end{array} .\right.
$$

If $k-n / p=1$ then the embedding holds for all $\alpha \in[0,1)$.

Our version is as in Ambrosetti and Malchiodi (2007). For a proof of a fairly more complete version, see Adams and Fournier (2008).

Theorem A.2.2. Let $\Omega \subset \mathbb{R}^{N}$ be a connected open set having the segment property and satisfying the cone condition, and let $k_{1}-k_{2}>N / 2$. Then the elements from $H^{k_{1}}(\Omega)$ are of class $C^{k_{2}}$ and the embedding

$$
H^{k_{1}}(\Omega) \hookrightarrow C^{k_{2}}(\bar{\Omega})
$$

is continuous.

For a proof and for a discussion of the segment and cone properties, see Wloka (1987). For us, it suffices to know that a smooth bounded open set satisfies both conditions.

Another important concept is that of the trace. See Evans (2010) or Kesavan (1989) for an introduction or Wloka (1987) for an extensive discussion.

Theorem A.2.3. Let $\Omega \subset \mathbb{R}^{N}$ be a bounded open set of class $C^{m+1}$. Then there exists continuous linear trace operator from $H^{m}(\Omega)$ into $\left(L^{2}(\partial \Omega)^{m}\right.$ such that

(i) If $v \in C^{\infty}(\bar{\Omega})$ then

$$
\gamma_{0}(v)=\left.v\right|_{\partial \Omega}, \gamma_{1}(v)=\left.\frac{\partial v}{\partial \boldsymbol{n}}\right|_{\partial \Omega}, \ldots, \gamma_{m-1}(v)=\left.\frac{\partial^{m} v}{\partial \boldsymbol{n}^{m}}\right|_{\partial \Omega}
$$

(ii) The range of $\gamma$ is the space

$$
\prod_{j=0}^{m-1} H^{m-j-1 / 2}(\partial \Omega)
$$

(iii) The kernel of $\gamma$ is $H_{0}^{m}(\Omega)$.

\section{A.3 The theorem of Borsuk-Ulam}

Theorem A.3.1. Let $\Omega \subset \mathbb{R}^{N}$ be an open, bounded and symmetric set containing the origin and $\varphi \in C\left(\partial \Omega, \mathbb{R}^{N}\right)$ and odd function such that $\varphi(\partial \Omega) \subset E$, where $E$ is an affine subspace of dimension less than or equal to $N-1$. Then there exists $x \in \partial \Omega$ such that $\varphi(x)=0$.

The proof depends on the theory of topological degree. See Kavian (1993) or Deimling (1985). 


\section{Bibliography}

R. A. Adams and J. J. F. Fournier. Sobolev Spaces. Academic Press, 2nd edition, 2008. 62

A. Ambrosetti and A. Malchiodi. Nonlinear Analysis and Semilinear Elliptic Problems. Cambridge University Press, 2007. 21, 27, 35, 62

A. Ambrosetti and G. Prodi. A Primer of Nonlinear Analysis. Cambridge University Press, 1993. 11,13

G. Bachman and L. Narici. Functional Analysis. Dover Publications, New York, 2000. 7

V. Benci and D. Fortunato. An eigenvalue problem for the Schrödinger-Maxwell equations. Topological Methods in Nonlinear Analysis, 11:283-293, 1998. 1, 52

P. Blanchard and E. Brüning. Variational Methods of Mathematical Physics. Springer, 1992. 11

A. Bonnet. A deformation lemma on a $C^{1}$ manifold. Manuscripta Mathematica, 81:339-359, 1993. 25

H. Brezis. Functional Analysis, Sobolev Spaces and Partial Differential Equations. Springer, New York, 2010. 4, 5

C. V. Coffman. A minimum-maximum principle for a class of nonlinear integral equations. Journal d'Analyse Mathématique, 22:391-419, 1969. 21

P. d'Avenia and G. Siciliano. Nonlinear Schrödinger equation in the Bopp-Podolsky electrodynamics: solutions in the electrostatic case. Journal of Differential Equations, 267: 1025-1065, 2019. 1

K. Deimling. Nonlinear Functional Analysis. Springer, 1985. 62

L. C. Evans. Partial Differential Equations, volume 19 of Graduate Studies in Mathematics. American Mathematical Society, 2nd edition, 2010. 62

F. Gazzola, H.-C. Grunau, and G. Sweers. Polyharmonic Boundary Value Problems. Springer, 2010. 50

O. Kavian. Introduction à la Theorie des Points Critiques. Springer, 1993. 21, 22, 62

S. Kesavan. Topics in Functional Analysis and Applications. Jhon Wiley \& Sons, 1989. 62

M. A. Krasnoselskii. Topological Methods in the Theory of Nonlinear Integral Equations. Macmillan, New York, 1964. 21

S. Lang. Differential and Riemannian Manifolds. Number 160 in Graduate Texts in Mathematics. Springer, 1995. 13 
S. Lang. Undergraduate Analysis. Undergraduate Texts in Mathematics. Springer, 2nd edition, 1997. 9, 11

R. S. Palais. Critical point theory and the minimax principle. Proc. Symp. Pure Math, 15: 185-212, 1970. 25

A. Pietsch. History of Banach Spaces and Linear Operators. Birkhäuser, Boston, 2007. ISBN 9780817643676.

L. Pisani and G. Siciliano. Neumann condition in the Scrhödinger-Maxwell system. Topological Methods in Nonlinear Analysis, 29:251-264, 2007. 60

L. Pisani and G. Siciliano. Constrained Schrödinger-Poisson system with non-constant interaction. Communications in Contemporary Mathematics, 15(1), 2013. 1

P. H. Rabinowitz. Some aspects of nonlinear eigenvalue problems. Rocky Mountain J. Math, 3:161-202, 1973. 21

P. H. Rabinowitz. Minimax Methods in Critical Point Theory with Applications to Differential Equations. American Mathematical Society, 1986.

M. Struwe. Variational Methods. Springer, 4th edition, 2008. 34, 36

A. Szulkin. Ljusternik-schnirelmann theory on $C^{1}$-manifolds. Annales de l'Institut Henri Poincaré, 1988. 36

M. E. Taylor. Partial Differential Equations I. Springer, 1996. 61

J. Wloka. Partial Differential Equations. Cambridge University Press, 1987. 62

E. Zeidler. Nonlinear Functional Analysis and its Applications, volume III. Springer, 1985.

E. Zeidler. Nonlinear Functional Analysis and its Applications, volume I. Springer, 1986. 19 


\section{Index}

Chain Rule, 5

constraint, 27

Deformation Lemma, 32

derivative, 4

Fréchet differentiable function, 3

Gateaux differentiable function, 6

Implicit Function Theorem, 18

Inverse Function Theorem, 16

locally invertible map, 15

Mean Value Inequality, 7

Minimax Principle, 35

Palais-Smale condition, 28

partial derivative, 12

second derivative, 10

sublevel, 25

tangent pseudo-gradient vector field, 29

Taylor's formula, 11

Theorem

Morse, 34 